\title{
Sparse Convex Clustering
}

\author{
Binhuan Wang㶲, Yilong Zhang ${ }^{2}$, Will Wei Sun ${ }^{3}$, Yixin Fang ${ }^{4}$ \\ ${ }^{1}$ New York University School of Medicine \\ ${ }^{2}$ Merck Research Laboratories \\ ${ }^{3}$ University of Miami School of Business Administration \\ ${ }^{4}$ Department of Mathematical Sciences, New Jersey Institute of Technology
}

\begin{abstract}
Convex clustering, a convex relaxation of k-means clustering and hierarchical clustering, has drawn recent attentions since it nicely addresses the instability issue of traditional nonconvex clustering methods. Although its computational and statistical properties have been recently studied, the performance of convex clustering has not yet been investigated in the high-dimensional clustering scenario, where the data contains a large number of features and many of them carry no information about the clustering structure. In this paper, we demonstrate that the performance of convex clustering could be distorted when the uninformative features are included in the clustering. To overcome it, we introduce a new clustering method, referred to as Sparse Convex Clustering, to simultaneously cluster observations and conduct feature selection. The key idea is to formulate convex clustering in a form of regularization, with an adaptive group-lasso penalty term on cluster centers. In order to optimally balance the trade-off between the cluster fitting and sparsity, a tuning criterion based on clustering stability is developed. Theoretically, we obtain a finite sample error bound for our estimator and further establish its variable selection consistency. The effectiveness of the proposed method is examined through a variety of numerical experiments and a real data application.
\end{abstract}

Key words: Convex clustering; Finite sample error; Group LASSO; High-dimensionality; Sparsity

\section{Introduction}

Cluster analysis is an unsupervised learning method and aims to assign observations into a number of clusters such that observations in the same group are similar to each other. Traditional clustering methods such as k-means clustering, hierarchical clustering, and Gaussian mixture models take a greedy approach and suffer from instabilities due to their non-convex optimization formulations.

To overcome the instability issues of these traditional clustering methods, a new clustering algorithm, Convex Clustering, has been recently proposed (Hocking et al., 2011; Lindsten et al., 2011; Pelckmans et al., 2005). Let $\mathbf{X} \in \mathbb{R}^{n \times p}$ be a data matrix with $n$ observations $X_{i}$,

${ }^{*}$ Correspondence to: 650 First Avenue Rm 578, New York, NY 10016; Email: Binhuan.Wang@ nyumc.org 
$i=1, \cdots, n$, and $p$ features. Convex clustering for these $n$ observations solves the following minimization problem:

$$
\min _{\mathbf{A} \in \mathbb{R}^{n \times p}} \frac{1}{2} \sum_{i=1}^{n}\left\|X_{i \cdot}-A_{i \cdot}\right\|_{2}^{2}+\gamma \sum_{i_{1}<i_{2}}\left\|A_{i_{1}} .-A_{i_{2}}\right\|_{q},
$$

where $A_{i}$. is the $i$-th row of $\mathbf{A}$ and $\|\cdot\|_{q}$ is the $L_{q}$-norm of a vector with $q \in\{1,2, \infty\}$. Note that both k-means clustering and hierarchical clustering consider $L_{0}$-norm in the second term, which leads to a non-convex optimization problem (Hocking et al., 2011; Tan and Witten, 2015). Therefore, convex clustering can be viewed as a convex relaxation of k-means clustering and hierarchical clustering, and the convex relaxation ensures that it achieves a unique global minimizer.

Due to the fused-lasso penalty (Tibshirani et al., 2005) in the second term of (1), the above formulation encourages that some of the rows of the solution $\widehat{\mathbf{A}}$ are identical. If $\widehat{A}_{i_{1}}$. $=\widehat{A}_{i_{2}}$, then observation $i_{1}$ and observation $i_{2}$ are said to belong to the same cluster. The tuning parameter $\gamma$ in $(1)$ controls the number of unique rows of $\widehat{\mathbf{A}}$, that is, the number of estimated clusters. When $\gamma=0, \widehat{\mathbf{A}}=\mathbf{X}$, and therefore each observation by itself is a cluster. As $\gamma$ increases, some of the rows of $\widehat{\mathbf{A}}$ become identical, which demonstrates a fusion process. For sufficiently large $\gamma$, all the rows of $\widehat{\mathbf{A}}$ will be identical, implying that all the observations are estimated to belong to a single cluster. Compared to traditional non-convex clustering methods, the solution $\widehat{\mathbf{A}}$ from convex clustering is unique for each given $\gamma$ since the objective function in (1) is strictly convex.

In recent years, the computational and statistical properties of convex clustering have been investigated. In particular, Zhu et al. (2014) provided conditions for convex clustering to recover the true clusters, Chi and Lange (2015) proposed efficient and scalable implementations for convex clustering, and Tan and Witten (2015) studied several statistical properties of convex clustering. While convex clustering enjoys nice theoretical properties and is computationally efficient, its performance can be severely deteriorated when clustering high-dimensional data where the number of features becomes large and many of them may contain no information about the clustering structure. Our extensive experimental studies demonstrate that in high-dimensional scenarios the 
performance of convex clustering is unsatisfactory when the uninformative features are included in the clustering. To overcome such a difficulty, a more appropriate convex clustering algorithm that can simultaneously perform cluster analysis and select informative variables is in demand.

In this article, we introduce a new clustering method, Sparse Convex Clustering, to incorporate the sparsity into convex clustering of high dimensional data. The key idea is to formulate convex clustering in a form of regularization, with an adaptive group-lasso penalty term on cluster centers to encourage the sparsity. Despite its simplicity, this regularization operator demands more challenging computational and statistical analysis than those in original convex clustering. In particular, computationally, we need to reformulate the sparse convex clustering into a few suboptimization problems and then solve each individual one via a pseudo regression formulation. To prove an unbiased estimator for the degrees of freedom of the proposed sparse convex clustering method, we need to carefully quantify the impact of variable selection due to the group lasso penalty. Moreover, we provide a non-asymptotic analysis for the prediction error of our sparse convex clustering estimator. Under a high-dimensional scenario where the dimension diverges with the sample size, our estimator is further shown to be consistent in variable selection. Note that our method is not only theoretical sound, but also practically promising. The superior performance of our procedure is demonstrated in extensive simulated examples and a real application of hand movement clustering.

We demonstrate the superior performance of the proposed method using a dataset generated from the fourth simulation setting in Section 5. In this dataset, there are 60 subjects from 4 clusters and 500 features, among which the first 20 features are informative. Figure 1 compares the performance of convex clustering (indicated as AMA on the left panel) and sparse convex clustering (indicated as S-AMA on the right panel), by visualizing the regularized feature matrix $\widehat{\mathbf{A}}$ estimated in each of the two methods. The heap maps show that sparse convex clustering screens out those uninformative features and therefore improves the clustering performance. 
Figure 1: The heat maps of $p \times n$ matrix $\widehat{\mathbf{A}}^{\mathrm{T}}$, estimated from Convex clustering and Sparse Convex clustering, respectively. Data are generated using Simulation Setting 4 where there are 60 subjects in 4 clusters and 500 features, among which the first 20 features are informative.

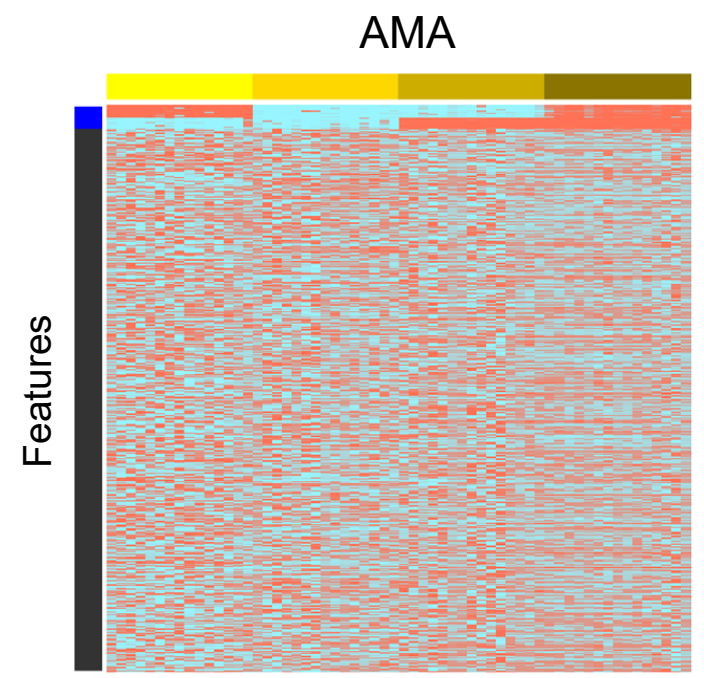

Subjects

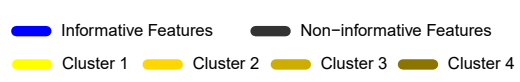

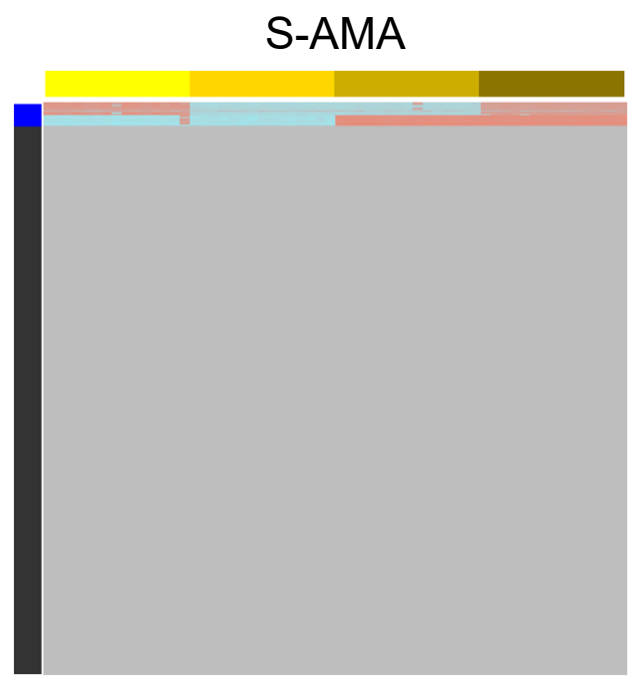

Subjects
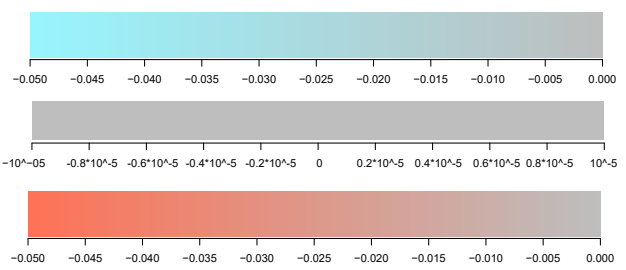

\subsection{Related Work}

A related paper on convex clustering is its efficient implementations proposed by Chi and Lange (2015) and its extension to convex biclustering has been developed by Chi et al. (2016). Two efficient algorithms ADMM and AMA are introduced while they are mainly designed for clustering low-dimensional data. In order to address high dimensionality, one key ingredient of our sparse convex clustering method is a new regularization penalty built upon their ADMM and AMA algorithms to encourage the sparsity structure of the clustering centers. As will be shown in experimental studies, such regularization step is able to significantly improve the clustering accuracy in high-dimensional clustering problems.

Another line of research focuses on simultaneous clustering and feature selection. Some ap- 
proaches are model-based clustering methods, such as Raftery and Dean (2006), Pan and Shen (2007), Wang and Zhu (2008), Xie et al. (2010), and Guo et al. (2010). In contrast, some approaches are model-free, such as Witten and Tibshirani (2010), Sun et al. (2012), and Wang et al. (2013). One common building block of these sparse clustering approaches is the usage of a lassotype penalty for feature selection. For example, Witten and Tibshirani (2010) developed a unified framework for feature selection in clustering using the lasso penalty (Tibshirani, 1996). Sun et al. (2012) proposed a sparse k-means using the group-lasso penalty (Yuan and Lin, 2006). We refer readers to Alelyani et al. (2013) for a thorough overview. In spite of their good numeric performance, these sparse clustering procedures still suffer from instabilities due to the non-convex optimization formulations. To overcome it, our sparse convex clustering solves a convex optimization problem and ensures a unique global solution.

\subsection{Paper Organization}

The rest of the manuscript is organized as follows. Section 2 introduces the sparse convex clustering as well as its two efficient algorithms. Section 3 studies its statistical properties and Section 4 discusses some practical issues in the proposed implementations. Section 5 evaluates the superior numeric performance of the proposed methods through extensive simulations and a real data application. Section 6 finishes this paper with a brief summary. Technical details are provided in Appendix or online supplementary.

\section{Sparse Convex Clustering}

This section presents the main results. We propose our new method, sparse convex clustering in Section 2.1, and then we develop two efficient algorithms to implement the method in Section 2.2

\subsection{Model}

To allow an adaptive penalization, we consider a modification of convex clustering (1), 


$$
\min _{\mathbf{A} \in \mathbb{R}^{n \times p}} \frac{1}{2} \sum_{i=1}^{n}\left\|X_{i}-A_{i} .\right\|_{2}^{2}+\gamma \sum_{i_{1}<i_{2}} w_{i_{1}, i_{2}}\left\|A_{i_{1}} \cdot-A_{i_{2}} .\right\|_{q}
$$

where the weight $w_{i_{1}, i_{2}} \geqslant 0$. Hocking et al. (2011) considered a pairwise affinity weight $w_{i_{1}, i_{2}}=$ $\exp \left(-\phi\left\|X_{i_{1}} \cdot-X_{i_{2}} \cdot\right\|_{2}^{2}\right)$ and Chi and Lange (2015) suggested $w_{i_{1}, i_{2}}=\iota_{i_{1}, i_{2}}^{m} \exp \left(-\phi\left\|X_{i_{1}} \cdot-X_{i_{2}} \cdot\right\|_{2}^{2}\right)$, where $\iota_{i_{1}, i_{2}}^{m}$ is 1 if observation $i_{2}$ is among $i_{1}$ 's $m$ nearest neighbors or vice verse, and 0 otherwise.

To introduce a reformulation of (2), we write the data matrix $\mathrm{X}$ in feature-level as column vector $\mathbf{X}=\left(\mathbf{x}_{1}, \cdots, \mathbf{x}_{p}\right)$, where $\mathbf{x}_{j}=\left(X_{1 j}, \cdots, X_{n j}\right)^{\mathrm{T}}, j=1, \ldots, p$ and denote $\mathbf{A}$ in featurelevel as column vector $\mathbf{A}=\left(\mathbf{a}_{1}, \cdots, \mathbf{a}_{p}\right)$. Without loss of generality, we assume the feature vectors are centered, i.e., $\sum_{i=1}^{n} X_{i j}=0$ for each $j=1, \ldots, p$. Simple algebra implies that (2) can be reformulated as

$$
\min _{\mathbf{A} \in \mathbb{R}^{n \times p}} \frac{1}{2} \sum_{j=1}^{p}\left\|\mathbf{x}_{j}-\mathbf{a}_{j}\right\|_{2}^{2}+\gamma \sum_{l \in \mathcal{E}} w_{l}\left\|A_{i_{1}}-A_{i_{2}} \cdot\right\|_{q}
$$

where $\mathcal{E}=\left\{l=\left(i_{1}, i_{2}\right): 1 \leqslant i_{1}<i_{2} \leqslant n\right\}$.

For a given $\gamma$, let $\widehat{\mathbf{A}}=\left(\widehat{A}_{1}, \cdots, \widehat{A}_{n .}\right)^{\mathrm{T}}=\left(\widehat{\mathbf{a}}_{1}, \ldots, \widehat{\mathbf{a}}_{p}\right)$ be the solution to 3 . The clustering structure is implied by the observation-level estimates, $\widehat{A}_{i}, i=1, \ldots, n$; that is, if $\widehat{A}_{i_{1}}$. $=\widehat{A}_{i_{2}}$, then observations $i_{1}$ and $i_{2}$ are estimated to belong to the same cluster. The feature importance is implied by the feature-level estimates, $\hat{\mathbf{a}}_{j}, j=1, \cdots, p$; that is, if the components of a featurelevel estimate $\widehat{\mathbf{a}}_{j}$ are identical, then the corresponding feature $j$ is not informative for clustering. Remind that the feature vectors are centered, then feature $j$ is not informative if and only if $\left\|\widehat{\mathbf{a}}_{j}\right\|_{2}^{2}=$ $\sum_{i=1}^{n} \widehat{A}_{i j}^{2}=0$.

In high-dimensional clustering, it is desired to have a sparse solution $\widehat{\mathbf{A}}$ with some of its column vectors being exact 0's. Motivated by the importance of excluding non-informative features, we propose a new sparse convex clustering by incorporating an adaptive group-lasso penalty (Wang and Leng, 2008; Yuan and Lin, 2006) into the convex clustering objective function (3). In particular, sparse convex clustering solves

$$
\min _{\mathbf{A} \in \mathbb{R}^{n \times p}} \frac{1}{2} \sum_{j=1}^{p}\left\|\mathbf{x}_{j}-\mathbf{a}_{j}\right\|_{2}^{2}+\gamma_{1} \sum_{l \in \mathcal{E}} w_{l}\left\|A_{i_{1}} \cdot-A_{i_{2}} .\right\|_{q}+\gamma_{2} \sum_{j=1}^{p} u_{j}\left\|\mathbf{a}_{j}\right\|_{2},
$$


where tuning parameter $\gamma_{1}$ controls the cluster size and tuning parameter $\gamma_{2}$ controls the number of informative features. In the group-lasso penalty, the weight $u_{j}$ plays an important role to adaptively penalize the features. Detailed discussions on practical choices of tuning parameters and weights can be found in Section 4 .

Remark 1 In the objective function (4) of sparse convex clustering, the second group-lasso-type penalty enforces the global sparsity condition; that is, the elements of each column vector $\mathbf{a}_{j}$ would be all zero or all nonzero. Such penalty is considered for the feature selection purpose. This global sparsity condition can be relaxed in two directions. First, we can replace the second penalty, $\gamma_{2} \sum_{j=1}^{p} u_{j}\left\|\mathbf{a}_{j}\right\|_{2}$, by a lasso type of penalty, $\gamma_{2} \sum_{j=1}^{p}\left\|\mathbf{a}_{j}\right\|_{1}$. Second, we can also add another penalty, $\sum_{j=1}^{p}\left\|\mathbf{a}_{j}\right\|_{1}$, to the objective function 4 . This results in a new penalty, $\gamma_{2} \sum_{j=1}^{p} u_{j}\left\|\mathbf{a}_{j}\right\|_{2}+$ $\gamma_{3} \sum_{j=1}^{p}\left\|\mathbf{a}_{j}\right\|_{1}$, which is the so-called sparse-group-lasso penalty (Friedman et al. 2010 .

\subsection{Algorithms}

This subsection discusses two efficient optimization approaches to solve the sparse convex clustering by adopting a similar computational strategy used in Chi and Lange (2015). Our two approaches are based on the alternating direction method of multipliers (ADMM) algorithm (Boyd et al., 2011; Gabay and Mercier, 1976, Glowinski and Marroco, 1975) and the alternating minimization algorithm (AMA) (Tseng, 1991), and are referred as sparse ADMM (S-ADMM) and sparse AMA (S-AMA), respectively.

To implement the S-ADMM and S-AMA algorithms, we rewrite the convex clustering problem in formula (4) as

$$
\begin{aligned}
\min _{\mathbf{A} \in \mathbb{R}^{n \times p}} & \frac{1}{2} \sum_{j=1}^{p}\left\|\mathbf{x}_{j}-\mathbf{a}_{j}\right\|_{2}^{2}+\gamma_{1} \sum_{l \in \mathcal{E}} w_{l}\left\|\mathbf{v}_{l}\right\|_{q}+\gamma_{2} \sum_{j=1}^{p} u_{j}\left\|\mathbf{a}_{j}\right\|_{2}, \\
\text { s.t. } & A_{i_{1}} \cdot-A_{i_{2}} .-\mathbf{v}_{l}=\mathbf{0} .
\end{aligned}
$$


This is equivalent to minimize the following augmented Lagrangian function,

$$
\begin{aligned}
\mathcal{L}_{\nu}(\mathbf{A}, \mathbf{V}, \boldsymbol{\Lambda})= & \frac{1}{2} \sum_{j=1}^{p}\left\|\mathbf{x}_{j}-\mathbf{a}_{j}\right\|_{2}^{2}+\gamma_{1} \sum_{l \in \mathcal{E}} w_{l}\left\|\mathbf{v}_{l}\right\|_{q}+\gamma_{2} \sum_{j=1}^{p} u_{i}\left\|_{\mathbf{a}_{j}}\right\|_{2} \\
& +\sum_{l \in \mathcal{E}}\left\langle\lambda_{l}, \mathbf{v}_{l}-A_{i_{1}}+A_{i_{2}}\right\rangle+\frac{\nu}{2} \sum_{l \in \mathcal{E}}\left\|\mathbf{v}_{l}-A_{i_{1}} .+A_{i_{2}} .\right\|_{2}^{2},
\end{aligned}
$$

where $\nu$ is a small constant, $\mathbf{V}=\left(\mathbf{v}_{1}, \ldots, \mathbf{v}_{|\mathcal{E}|}\right)$, and $\boldsymbol{\Lambda}=\left(\lambda_{1}, \ldots, \lambda_{|\mathcal{E}|}\right)$. Compared with the original algorithms proposed in Chi and Lange (2015), it becomes challenging to deal with the feature-level and observation-level vectors in the new objective function simultaneously.

\subsubsection{S-ADMM}

S-ADMM minimizes the augmented Lagrangian problem by alternatively solving one block of variables at a time. In particular, S-ADMM solves

$$
\begin{aligned}
\mathbf{A}^{m+1} & =\underset{\mathbf{A}}{\operatorname{argmin}} \mathcal{L}_{\nu}\left(\mathbf{A}, \mathbf{V}^{m}, \boldsymbol{\Lambda}^{m}\right), \\
\mathbf{V}^{m+1} & =\underset{\mathbf{V}}{\operatorname{argmin}} \mathcal{L}_{\nu}\left(\mathbf{A}^{m+1}, \mathbf{V}, \mathbf{\Lambda}^{m}\right), \\
\boldsymbol{\lambda}_{l}^{m+1} & =\boldsymbol{\lambda}_{l}^{m}+\nu\left(\mathbf{v}_{l}^{m+1}-A_{i_{1} .}^{m+1}+A_{i_{2} \cdot}^{m+1}\right), l \in \mathcal{E} .
\end{aligned}
$$

Next we discuss the detailed updating implementations for $\mathbf{A}, \mathbf{V}$ and $\boldsymbol{\Lambda}$ in three steps. $\mathrm{A}$ summary of the S-ADMM algorithm is shown in Algorithm 1

Step 1: update A. Denote $\widetilde{\mathbf{v}}_{l}=\mathbf{v}_{l}+\frac{1}{\nu} \boldsymbol{\lambda}_{l}$. Updating $\mathbf{A}$ is equivalent to minimizing

$$
f(\mathbf{A})=\frac{1}{2} \sum_{j=1}^{p}\left\|\mathbf{x}_{j}-\mathbf{a}_{j}\right\|_{2}^{2}+\frac{\nu}{2} \sum_{l \in \mathcal{E}}\left\|\widetilde{\mathbf{v}}_{l}-A_{i_{1}}+A_{i_{2}}\right\|_{2}^{2}+\gamma_{2} \sum_{j=1}^{p} u_{j}\left\|\mathbf{a}_{j}\right\|_{2}
$$

This optimization problem is challenging because the objective function involves both rows and columns of the matrix A. To tackle this difficulty, the following key lemma associates (6) with a group-lasso regression problem which can be efficiently solved via standard packages.

Lemma 1 Let $\mathbf{I}_{n}$ be an $n \times n$ identity matrix, $\mathbf{1}_{n}$ be an $n$-dimensional vector with each component 
being 1 , and $\mathbf{e}_{i}$ be an $n$-dimensional vector with each component being 0 but its $i$-th component being 1. Define $\mathbf{N}^{-1}=(1+n \nu)^{-1 / 2}\left[\mathbf{I}_{n}+n^{-1}(\sqrt{1+n \nu}-1) \mathbf{1}_{n} \mathbf{1}_{n}^{\mathrm{T}}\right]$ and denote $\mathbf{y}_{j}=\mathbf{N}^{-1}\left[\mathbf{x}_{j}+\right.$ $\left.\nu \sum_{l \in \mathcal{E}} \widetilde{v}_{j l}\left(\mathbf{e}_{i_{1}}-\mathbf{e}_{i_{2}}\right)\right]$ with $\widetilde{v}_{j l}$ the $j$-th element of $\widetilde{\mathbf{v}}_{l}$. Then, minimizing (6) is equivalent to

$$
\min _{\mathbf{a}_{j}} \frac{1}{2}\left\|\mathbf{y}_{j}-\mathbf{N a}_{j}\right\|_{2}^{2}+\gamma_{2} u_{j}\left\|\mathbf{a}_{j}\right\|_{2}, \text { for each } j=1, \ldots, p \text {. }
$$

The proof of Lemma 1 is discussed in Appendix. The key ingredient in the proof is a newly established property of a permutation matrix, i.e., Proposition 1. Based on this property, we are able to solve the minimization of $f(\mathbf{A})$ by $p$ separate sub-optimization problems. This together with the property of group-lasso penalty leads to desirable results. Recall that we require the feature vectors are centered, so we center corresponding estimates during each iteration.

Step 2: update V. For any $\sigma>0$ and norm $\Omega(\cdot)$, we define a proximal map,

$$
\operatorname{prox}_{\sigma \Omega}(\mathbf{u})=\underset{\mathbf{v}}{\operatorname{argmin}}\left[\sigma \Omega(\mathbf{v})+\frac{1}{2}\|\mathbf{u}-\mathbf{v}\|_{2}^{2}\right]
$$

In S-ADMM, $\Omega(\cdot)$ is a $q$-norm $\|\cdot\|_{q}$ with $q=1,2$, or $\infty$, and $\sigma=\gamma_{1} w_{l} / \nu$. We refer the readers to Table 1 of Chi and Lange (2015) for the explicit formulations of the proximal map of $q$-norm for $q=1,2$ and $\infty$. Because vectors $\mathbf{v}_{l}$ are separable, they can be solved via proximal maps, that is

$$
\begin{aligned}
\mathbf{v}_{l} & =\underset{\mathbf{v}_{l}}{\operatorname{argmin}} \frac{1}{2}\left\|\mathbf{v}_{l}-\left(A_{i_{1}} \cdot-A_{i_{2} \cdot}-\nu^{-1} \boldsymbol{\lambda}_{l}\right)\right\|_{2}^{2}+\frac{\gamma_{1} w_{l}}{\nu}\left\|\mathbf{v}_{l}\right\|_{q} \\
& =\operatorname{prox}_{\sigma_{l}\|\cdot\|_{q}}\left(A_{i_{1}}-A_{i_{2}} \cdot-\nu^{-1} \boldsymbol{\lambda}_{l}\right) .
\end{aligned}
$$

Step 3: update $\Lambda$. Finally, $\boldsymbol{\lambda}_{l}$ can be updated by $\boldsymbol{\lambda}_{l}=\boldsymbol{\lambda}_{l}+\nu\left(\mathbf{v}_{l}-A_{i_{1}}\right.$. $+A_{i_{2}}$. $)$.

\subsubsection{S-AMA}

To increase the computational efficiency, we introduce another algorithm S-AMA for implementing sparse convex clustering. S-AMA is different from S-ADMM in the update of A. In particular, S-AMA solves $\mathbf{A}$ by treating $\nu=0$, i.e., $\mathbf{A}^{m+1}=\operatorname{argmin}_{\mathbf{A}} \mathcal{L}_{0}\left(\mathbf{A}, \mathbf{V}^{m}, \boldsymbol{\Lambda}^{m}\right)$. When $\nu=0$, we 


\section{Algorithm 1 S-ADMM}

1. Initialize $\mathbf{V}^{0}$ and $\boldsymbol{\Lambda}^{0}$. For $m=1,2, \ldots$

2. For $j=1, \ldots, p$, do

$$
\begin{aligned}
\widetilde{\mathbf{v}}_{l}^{m-1} & =\mathbf{v}_{l}^{m-1}+\frac{1}{\nu} \boldsymbol{\lambda}_{l}^{m-1}, l \in \mathcal{E} \\
\mathbf{y}_{j}^{m-1} & =\mathbf{N}^{-1}\left(\mathbf{x}_{j}+\nu \sum_{l \in \mathcal{E}} \widetilde{v}_{l j}^{m-1}\left(\mathbf{e}_{i_{1}}-\mathbf{e}_{i_{2}}\right)\right), \\
\mathbf{a}_{j}^{m} & =\underset{\mathbf{a}_{j}}{\operatorname{argmin}} \frac{1}{2}\left\|\mathbf{y}_{j}^{m-1}-\mathbf{N} \mathbf{a}_{j}\right\|_{2}^{2}+\gamma_{2} u_{j}\left\|\mathbf{a}_{j}\right\|_{2}, \\
\mathbf{a}_{j}^{m} & =\mathbf{a}_{j}^{m}-\overline{\mathbf{a}}_{j}^{m} \mathbf{1}_{n}, \text { where } \overline{\mathbf{a}}_{j}^{m}=\mathbf{1}_{n}^{T} \mathbf{a}_{j}^{m} / n .
\end{aligned}
$$

3. For $l \in \mathcal{E}$, do

$$
\mathbf{v}_{l}^{m}=\operatorname{prox}_{\sigma_{l}\|\|_{q}}\left(A_{i_{1} \cdot}^{m}-A_{i_{2} \cdot}^{m}-\nu^{-1} \boldsymbol{\lambda}_{l}^{m-1}\right)
$$

4. For $l \in \mathcal{E}$, do

$$
\boldsymbol{\lambda}_{l}^{m}=\boldsymbol{\lambda}_{l}^{m-1}+\nu\left(\mathbf{v}_{l}^{m}-A_{i_{1}}^{m}+A_{i_{2}}^{m}\right)
$$

5. Repeat Steps 2-4 until convergence.

have $\mathbf{N}=\mathbf{I}_{n}$ and $\mathbf{y}_{j}=\mathbf{x}_{j}$. According to Lemma 1, updating $\mathbf{A}$ requires to solve $p$ group-lasso problems:

$$
\min _{\mathbf{a}_{j}} \frac{1}{2}\left\|\mathbf{x}_{j}-\mathbf{a}_{j}\right\|_{2}^{2}+\gamma_{2} u_{j}\left\|\mathbf{a}_{j}\right\|_{2}, j=1, \ldots, p .
$$

By Karush-Kuhn-Tucker (KKT) conditions of the group lasso problem (Yuan and Lin, 2006), the solution to (7) has a closed form as

$$
\widehat{\mathbf{a}}_{j}=\left(1-\frac{\gamma_{2} u_{j}}{\left\|\mathbf{z}_{j}\right\|_{2}}\right)_{+} \mathbf{z}_{j}
$$

where $\mathbf{z}_{j}=\mathbf{x}_{j}+\sum_{l \in \mathcal{E}} \lambda_{j l}\left(\mathbf{e}_{i_{1}}-\mathbf{e}_{i_{2}}\right)$ and $(z)_{+}=\max \{0, z\}$. See the detailed derivations in online Supplementary. Still, we center $\widehat{\mathbf{a}}_{j}$ for each $j$. The above formula significantly reduces the computational cost by solving $p$ group-lasso problem analytically in each iteration. Note that the above update of $\mathbf{A}$ is independent of $\mathbf{V}$, which indicates that S-AMA algorithm does not need to 
compute the update of $\mathrm{V}$. Therefore S-AMA is much more efficient than S-ADMM algorithm.

Next, we discuss the update of $\Lambda$. Define $\mathcal{P}_{B}(\mathbf{z})$ as a projection onto $B=\left\{\mathbf{y}:\|\mathbf{y}\|_{\dagger} \leqslant 1\right\}$ of the norm $\|\cdot\|_{\dagger}$, where $\|\cdot\|_{\dagger}$ is the dual norm of $\|\cdot\|_{q}$, which defines the fusion penalty. We show in online Supplementary that the update of $\boldsymbol{\Lambda}$ reduces to $\boldsymbol{\lambda}_{l}^{m}=\mathcal{P}_{C_{l}}\left[\boldsymbol{\lambda}_{l}^{m-1}-\nu\left(A_{i_{1}}^{m}\right.\right.$. $-A_{i_{2}}^{m}$. $\left.)\right]$ with $C_{l}=\left\{\boldsymbol{\lambda}_{l}:\left\|\boldsymbol{\lambda}_{l}\right\|_{\dagger} \leqslant \gamma_{1} w_{l}\right\}$. The S-AMA algorithm is summarized in Algorithm 2 .

\section{Algorithm 2 S-AMA}

1. Initialize $\Lambda^{0}$. For $m=1,2, \ldots$

2. For $j=1, \ldots, p$, do

$$
\begin{aligned}
\mathbf{z}_{j}^{m} & =\mathbf{x}_{j}+\sum_{l \in \mathcal{E}} \lambda_{l j}^{m-1}\left(\mathbf{e}_{i_{1}}-\mathbf{e}_{i_{2}}\right), \\
\mathbf{a}_{j}^{m} & =\left(1-\frac{\gamma_{2} u_{i}}{\left\|\mathbf{z}_{i}^{m}\right\|_{2}}\right)_{+} \mathbf{z}_{j}^{m}, \\
\mathbf{a}_{j}^{m} & =\mathbf{a}_{j}^{m}-\overline{\mathbf{a}}_{j}^{m} \mathbf{1}_{n}, \text { where } \overline{\mathbf{a}}_{j}^{m}=\mathbf{1}_{n}^{T} \mathbf{a}_{j}^{m} / n .
\end{aligned}
$$

3. For $l \in \mathcal{E}$, do

$$
\boldsymbol{\lambda}_{l}^{m}=\mathcal{P}_{C_{l}}\left[\boldsymbol{\lambda}_{l}^{m-1}-\nu\left(A_{i_{1} \cdot}^{m}-A_{i_{2}}^{m}\right)\right],
$$

where $C_{l}=\left\{\boldsymbol{\lambda}_{l}:\left\|\boldsymbol{\lambda}_{l}\right\|_{\dagger} \leqslant \gamma_{1} w_{l}\right\}$.

4. Repeat Steps 2-3 until convergence.

\subsubsection{Algorithmic Convergence}

This subsection discusses the convergence of the proposed S-ADMM and S-AMA algorithms. Chi and Lange (2015) and the references therein provided sufficient conditions for the convergence of the following general optimization problem,

$$
\min _{\xi, \zeta} f(\xi)+g(\zeta), \text { s.t. } A \xi+B \zeta=c
$$

They verified that the ADMM and AMA algorithms for convex clustering, as two special cases of (8), satisfied the sufficient conditions under which the convergence was guaranteed.

The convergence of our S-ADMM and S-AMA algorithms follows similar arguments. Note 
that the only difference between the objective function in (4) and its counterpart in Chi and Lange (2015) is a convex penalty term $\gamma_{2} \sum_{j=1}^{p} u_{j}\left\|\mathbf{a}_{j}\right\|_{2}$. Define the summation of the first and third terms of the objective function in (4) as $f(\cdot)$, and the second term as $g(\cdot)$. This indicates that the optimization problem (4) is a special case of (8). Simple algebra implies that $f(\cdot)$ is strongly convex. According to Chi and Lange (2015), one can show that, under mild regularization conditions, the convergence of S-ADMM is guaranteed for any $\nu>0$, and the convergence of S-AMA algorithm is guaranteed provided that positive constant $\nu$ is not too large.

\subsubsection{Computational Consideration}

Step 2 in both Algorithms 1 and 2 involves $p$ sub-optimization problems. Therefore, S-ADMM and S-AMA merit from the distributed optimization, and they can handle large-scale problems efficiently. To be specific, Step 2 can be distributed to different processors to obtain estimates of $\mathbf{a}_{j}$ 's which are then gathered to update A. In addition, Steps 3-4 in Algorithm 1 or Step 3 in Algorithm 2 can also be distributed to different processors to obtain fast updates.

It is worth pointing out that the computation of S-AMA is comparable to AMA in Chi and Lange (2015), while S-ADMM is computationally more expensive than ADMM in Chi and Lange (2015) and S-AMA. This is because Step 2 in S-ADMM does not have a closed-form formula and it requires solving $p$ group-lasso problems assisted by iterations. Furthermore, Step 3 in S-AMA only requires updates for $\boldsymbol{\lambda}_{l}$ for those $l$ such that $w_{l}>0, l \in \mathcal{E}$. With a suitable selection of $w_{l}$ discussed in Section 4.1, the size of working set of $l$ can be dramatically reduced from $n(n-1) / 2$ to a much smaller number. Our limited experience in numerical studies also confirms the superiority of S-AMA over S-ADMM in terms of the computational cost.

We have developed an R package "scvxclustr" to facilitate the implementation of proposed methods. Table 1 compares the computational time in seconds of our package with Chi and Lange (2015)'s method via "cvxclustr" for the setting with 4 clusters described in Section 5.1 with given tuning parameters. The computer is equipped with a CPU i3-4170 $(3.70 \mathrm{GHz})$ and $8 \mathrm{G}$ memory. 
Table 1: Timing comparison under the 4 cluster setting described in Section 5.1 with given tuning parameters under various combinations of $n$ and $p$. The time is in second.

\begin{tabular}{|c|c|c|c|c|c|c|c|c|c|c|c|c|}
\hline$n$ & \multicolumn{3}{|c|}{60} & \multicolumn{3}{|c|}{120} & \multicolumn{3}{|c|}{300} & \multicolumn{3}{|c|}{400} \\
\hline$p$ & 150 & 300 & 500 & 150 & 300 & 500 & 150 & 300 & 500 & 150 & 300 & 500 \\
\hline AMA & 0.6 & 3 & 5 & 3 & 7 & 13 & 11 & 23 & 37 & 15 & 32 & 51 \\
\hline ADMM & 6 & 17 & 24 & 19 & 71 & 82 & 157 & 375 & 548 & 329 & 1482 & 1796 \\
\hline S-AMA & 0.1 & 0.6 & 2 & 0.8 & 2 & 6 & 3 & 10 & 25 & 6 & 17 & 34 \\
\hline S-ADMM & 16 & 47 & 60 & 60 & 152 & 215 & 593 & 2051 & 2781 & 1252 & 3639 & 7925 \\
\hline
\end{tabular}

\section{Theoretical Properties}

In this section, we study statistical properties of the proposed sparse convex clustering. In particular, we provide finite sample bounds for prediction error of the proposed sparse convex clustering estimator and establish its variable selection consistency. In Section S.3 of online Supplementary, we also develop unbiased estimators for the degrees of freedoms of sparse convex clustering.

Assume $\mathbf{x}=\mathbf{a}_{0}+\varepsilon$, where $\varepsilon \in \mathbb{R}^{n p}$ is a vector of independent sub-Gaussian noise terms with mean zero and variance $\sigma^{2}$, and $\mathbf{a}_{0}=\operatorname{vec}\left(\mathbf{A}_{0}\right)=\left(\mathbf{a}_{01}^{\mathrm{T}}, \ldots, \mathbf{a}_{0 p}^{\mathrm{T}}\right)^{\mathrm{T}}$ is a $n p$-dimensional mean vector. Without loss of generality, we assume that only the first $p_{0}<p$ features are informative, i.e., $\left\|\mathbf{a}_{0 j}\right\|_{2} \neq 0$ for $j \leqslant p_{0}$ and $\left\|\mathbf{a}_{0 j}\right\|_{2}=0$ for $j>p_{0}$. The informative feature set is denoted as $\mathcal{A}=\left\{1, \ldots, p_{0}\right\}$ and the noninformative feature set is $\mathcal{A}^{c}=\left\{p_{0}+1, \ldots, p\right\}$. For simplicity, we consider the case with $w_{l}=1$.

Our sparse convex clustering in (4) can be reformulated as the following problem:

$$
\widehat{\mathbf{a}}=\underset{\mathbf{a} \in \mathbb{R}^{n p}}{\operatorname{argmin}} \frac{1}{2}\|\mathbf{x}-\mathbf{a}\|_{2}^{2}+\gamma_{1} \sum_{l \in \mathcal{E}}\left\|\mathbf{C}_{l} \mathbf{a}\right\|_{q}+\gamma_{2} \sum_{j=1}^{p} u_{j}\left\|\mathbf{a}_{j}\right\|_{2},
$$

where $\mathbf{C}_{l}=\mathbf{I}_{p} \otimes\left(\mathbf{e}_{i_{1}}-\mathbf{e}_{i_{2}}\right)^{\mathrm{T}}$ and hence $\mathbf{C}_{l} \mathbf{a}=A_{i_{1}}$. $-A_{i_{2}}$. Define $\mathbf{C}=\left(\mathbf{C}_{1}^{\mathrm{T}}, \ldots, \mathbf{C}_{|\mathcal{E}|}^{\mathrm{T}}\right)^{\mathrm{T}}$ and denote $\mathbf{u}=\left(u_{1}, \ldots, u_{p}\right)^{\mathrm{T}}$.

The following two theorems provide the finite sample bounds for prediction error of our sparse convex clustering estimator with parameter $q \in\{1,2\}$, respectively. 
Theorem 1 Let $\hat{\mathbf{a}}$ be the estimate of (9) with $q=1$. If $\gamma_{1}>4 \sigma \sqrt{\frac{\log \left(p \cdot\left(\begin{array}{l}n \\ 2\end{array}\right)\right)}{n}}$, then

$$
\frac{1-\gamma_{2}}{2 n p}\left\|\widehat{\mathbf{a}}-\mathbf{a}_{0}\right\|_{2}^{2} \leqslant \frac{3 \gamma_{1}}{2 n p}\left\|\mathbf{C} \mathbf{a}_{0}\right\|_{1}+\frac{\gamma_{2}\|\mathbf{u}\|_{2}^{2}}{2 n p}+\sigma^{2}\left[\frac{1}{n}+\sqrt{\frac{\log (n p)}{n^{2} p}}\right]+\frac{1}{n p}
$$

holds with probability at least $1-c_{3}$, where

$$
c_{3}=\frac{2}{p \cdot\left(\begin{array}{l}
n \\
2
\end{array}\right)}+\exp \left\{-\min \left(c_{1} \log (n p), c_{2} \sqrt{p \log (n p)}\right)\right\}+2 \exp \left(-n p /\left(2 \sigma^{2} \gamma_{2}^{2}\|\mathbf{u}\|_{1}^{2}\right)\right)
$$

for some positive constants $c_{1}$ and $c_{2}$ defined in Lemma S.1.

Theorem 2 Let $\hat{\mathbf{a}}$ be the estimate of (9) with $q=2$. If $\gamma_{1}>4 \sigma \sqrt{\frac{\log \left(p \cdot\left(\begin{array}{l}n \\ 2\end{array}\right)\right)}{n}}$, then

$$
\frac{1-\gamma_{2}}{2 n p}\left\|\widehat{\mathbf{a}}-\mathbf{a}_{0}\right\|_{2}^{2} \leqslant \frac{3 \gamma_{1}}{2 n p} \sum_{l \in \mathcal{E}}\left\|\mathbf{C}_{l} \mathbf{a}_{0}\right\|_{2}+\frac{\gamma_{2}\|\mathbf{u}\|_{2}^{2}}{2 n p}+\sigma^{2}\left[\frac{1}{n}+\sqrt{\frac{\log (n p)}{n^{2} p}}\right]+\frac{1}{n p}
$$

holds with probability at least $1-c_{3}$, where $c_{3}$ is defined in Theorem 1

Remark 2 Based on the sparsity assumption of features, we know true underlying clusters differ only with respect to the first $p_{0}$ features. Thus, $\left\|\mathbf{C}_{l} \mathbf{a}_{0}\right\|_{1}=O(1)$ and $\left\|\mathbf{C}_{l} \mathbf{a}_{0}\right\|_{2}=O(1), \forall l \in \mathcal{E}$. Note that $|\mathcal{E}|=\left(\begin{array}{l}n \\ 2\end{array}\right)$, and hence $\left\|\mathbf{C a}_{0}\right\|_{1}=O\left(n^{2}\right)$ and $\sum_{l \in \mathcal{E}}\left\|\mathbf{C}_{l} \mathbf{a}_{0}\right\|_{2}=O\left(n^{2}\right)$. In order to obtain a general prediction consistency based on Theorems 1,2 we need $\gamma_{2} \rightarrow 0, c_{3} \rightarrow 0$ and the first two terms on right-hand side of the inequalities in Theorems $1 / 2$ vanish. Assume $\gamma_{1}\left\|\mathbf{C a}_{0}\right\|_{1} /(2 n p)=$ $o(1)$, and then we have $\sqrt{n \log \left(p \cdot\left(\begin{array}{l}n \\ 2\end{array}\right)\right) / p^{2}}=o(1)$. Additionally, $c_{3} \rightarrow 0$ and $\gamma_{2}\|\mathbf{u}\|_{2}^{2} /(2 n p)$ are equivalent to requiring $\gamma_{2}\|\mathbf{u}\|_{1}^{2} /(n p) \rightarrow 0$ by noting that $\|\mathbf{u}\|_{2} \leqslant\|\mathbf{u}\|_{1}$.

In particular, we discuss following two choices for the weight $u_{j}$, which lead to prediction consistency. First, we assume a non-adaptive weight, i.e., $u_{j} \equiv 1$. It is easy to see $\|\mathbf{u}\|_{1}=p$. Additionally, we require $\gamma_{2} \rightarrow 0$ and $\gamma_{2} p n^{-1} \rightarrow 0$. Second, more generally, we assume $\|\mathbf{u}\|_{1}$ is bounded above by $p^{\zeta}$, where $\zeta$ is a constant, and require $\gamma_{2} \rightarrow 0, \gamma_{2} p^{2 \zeta-1} n^{-1} \rightarrow 0$. Therefore, in either case $\gamma_{2}\|\mathbf{u}\|_{1}^{2} /(n p) \rightarrow 0$ and then $\hat{\mathbf{a}}$ is prediction consistent with $q=1$ or $q=2$.

Next, we establish the asymptotic selection consistency of the proposed sparse convex cluster- 
ing, which is a desirable property in high-dimensional cluster analysis where many features are non-informative.

Theorem 3 If $\gamma_{1}>4 \sigma \sqrt{\log \left(p \cdot\left(\begin{array}{c}n \\ 2\end{array}\right)\right) / n}, \gamma_{1}\left\|\mathbf{C a}_{0}\right\|_{1} /(2 n p)=o(1), \gamma_{2} \rightarrow 0$ and $\gamma_{2}\|\mathbf{u}\|_{1}^{2} /(n p) \rightarrow 0$ as $n, p \rightarrow \infty$, then $P\left(\left\|\widehat{\mathbf{a}}_{j}\right\|_{2}=0\right) \rightarrow 1$ for any $j \in \mathcal{A}^{c}$, with the solution $\hat{\mathbf{a}}$ to $(9)$ with either $q=1$ or $q=2$.

Remark 3 Condition $\gamma_{2}\|\mathbf{u}\|_{1}^{2} /(n p) \rightarrow 0$ generally implies that the adaptive weights cannot be too large. For example, uniform weights satisfy this condition. Note that Conditions $\gamma_{1}>$ $4 \sigma \sqrt{\log \left(p \cdot\left(\begin{array}{l}n \\ 2\end{array}\right)\right) / n}$ and $\gamma_{1}\left\|\mathbf{C a}_{0}\right\|_{1} /(2 n p)=o(1)$ imply $\sqrt{n \log \left(p \cdot\left(\begin{array}{l}n \\ 2\end{array}\right)\right) / p^{2}}=o(1)$. This is derived to ensure the estimation consistency in Theorems 1 and 2 in particular, to ensure the first term on the right-hand side of Theorem 1 or 2 to converge to zero. This condition requires that $n=o\left(p^{2}\right)$ up to a log-term, and hence is satisfied as long as p diverges not too slow, which is typically true in high-dimensional scenarios. Note that in this case p can still be smaller than $n$. Similar phenomenon has also been found in Tan and Witten (2015).

Theorem 3 establishes the asymptotic selection consistency in the sense that the proposed sparse convex clustering can eliminate the non-informative variables in the estimated cluster centers with probability tending to one.This variable selection consistency is illustrated in the motivation example shown in Figure 1.

Proofs of Theorems 1,3 are provided in online Supplementary.

\section{Practical Issues}

In Section 2.2, the S-ADMM and S-AMA algorithms rely on the choice of weights and the tuning parameters $\gamma_{1}$ and $\gamma_{2}$. In this section, we discuss how to choose these parameters in practice. 


\subsection{Selection of Weights}

This subsection introduces practical selections of the weights $w_{i_{1}, i_{2}},\left(i_{1}, i_{2}\right) \in \mathcal{E}$, in the fused-lasso penalty, and the factors $u_{j}, j=1, \cdots, p$, in the adaptive group-lasso penalty.

Following Chi and Lange (2015), we choose weights by incorporating the m-nearest-neighbors method with Gaussian kernel. To be specific, the weight between the sample pair $\left(i_{1}, i_{2}\right)$ is set as $w_{i_{1}, i_{2}}=\iota_{i_{1}, i_{2}}^{m} \exp \left(-\phi\left\|X_{i_{1}} \cdot-X_{i_{2}}\right\|_{2}^{2}\right)$, where $\iota_{i_{1}, i_{2}}^{m}$ equals 1 if observation $i_{2}$ is among observation $i_{1}$ 's $m$ nearest neighbors or vice versa, and 0 otherwise. This choice of weights works well for a wide range of $\phi$ when $m$ is small. In our numerical results, $m$ is fixed at 5 and $\phi$ is fixed at 0.5 .

Next we consider the selection of factor $u_{j}$. As suggested by Zou (2006), $u_{j}$ can be chosen as $1 /\left\|\widehat{\mathbf{a}}_{j}^{(0)}\right\|_{2}$, where $\hat{\mathbf{a}}_{j}^{(0)}$ is the estimate of $\mathbf{a}_{j}$ in (4) with $\gamma_{2}=0$. Such choice of factors penalizes less on informative features and penalizes more on uninformative features, and hence leads to improved clustering accuracy and variable selection performance than its non-adaptive counterpart.

Finally, in order to ensure that the optimal tuning parameters $\gamma_{1}$ and $\gamma_{2}$ lie in relatively robust intervals regardless of feature dimension and sample size, weights $w_{i_{1}, i_{2}}$ and factors $u_{j}$ are rescaled to sum to $1 / \sqrt{p}$ and $1 / \sqrt{n}$, respectively. Such re-scaling is only for convenience and does not affect the final clustering path.

\subsection{Selection of Tuning Parameters}

This subsection provides a selection method for tuning parameters $\gamma_{1}$ and $\gamma_{2}$. Remind that $\gamma_{1}$ controls the number of estimated clusters and $\gamma_{2}$ controls the number of selected informative features.

We first illustrate via a toy example the effectiveness of tuning parameter $\gamma_{2}$ on variable selection accuracy. In this example, 60 observations with $p=500$ features are generated from 4 clusters. Among all the features, only 20 variables differ between clusters. See detailed simulation setup in Section 5.1. By fixing $\widehat{\gamma}_{1}=2.44$ and varying $\gamma_{2}$ from $e^{-5.0}$ to $e^{7.0}$, we plot the path of false negative rate (FNR) and the path of false positive rate (FPR) of the final estimator. As shown in Figure 2, when $\gamma_{2}$ is close to zero, all features are included, and when $\gamma_{2}$ increases to some ranges of intervals, all and only uninformative features are excluded, i.e., perfect variable selection 
performance. This illustrates the sensitivity of $\gamma_{2}$ to the variable selection performance of the final estimator. In practice, we aim to estimate a suitable $\gamma_{2}$ that leads to satisfactory variable selection.

Figure 2: Illustration of the effectiveness of $\gamma_{2}$ on variable selection accuracy. The solid curve is the path of false negative rate (FNR), and the dashed curve is the path of false positive rate (FPR).

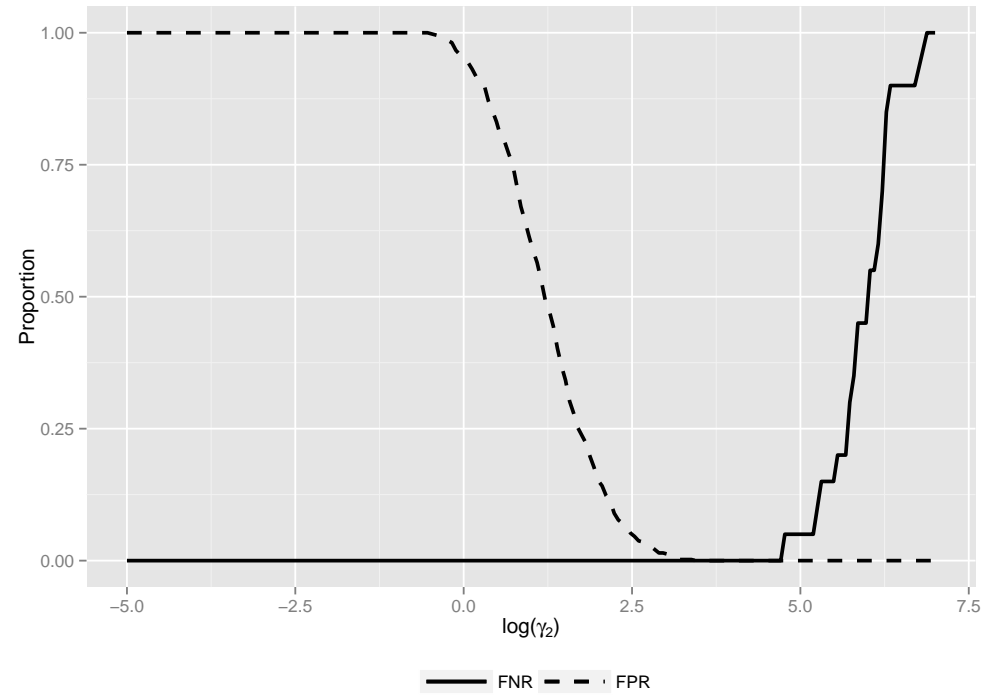

In literature, Wang (2010) and Fang and Wang (2012) proposed stability selection to estimate the tuning parameters in clustering models. The idea behind stability selection is that a good tuning parameter should produce clustering results that are stable with respect to a small perturbation to the training samples. Stability selection well suits the model selection in cluster analysis because cluster labels are unavailable and the cross-validation method is not applicable in this case.

In this paper, we propose to use stability selection in Fang and Wang (2012) to tune both parameters $\gamma_{1}$ and $\gamma_{2}$. To be specific, for any given $\gamma_{1}$ and $\gamma_{2}$, based on two sets of bootstrapped samples, two clustering results can be produced via (4), and then the stability measurement (Fang and Wang, 2012) can be computed to measure the agreement between the two clustering results. In order to enhance the robustness of the stability selection method, we repeat this procedure 50 times and then compute the averaged stability value. Finally, the optimal parameter is selected as the one achieving maximum stability. Our extensive numerical studies show that the selection of important features is less sensitive to the clustering path, i.e., import features stand out in almost all clustering structures. Thus, to speed up tuning process, stability path can be computed over of 
a coarse grid of $\gamma_{1}$ and a fine grid of $\gamma_{2}$.

\section{Numerical Results}

This section demonstrates the superior performance of our sparse convex clustering in simulated examples in Section 5.1 and a real application of hand movement clustering in Section 5.2 .

\subsection{Simulation Studies}

In this subsection, simulations studies are conducted to evaluate the performance of sparse convex clustering methods (S-ADMM and S-AMA). They are compared to the k-means clustering and two convex clustering algorithms: ADMM and AMA (Chi and Lange, 2015).

First, we consider four spherical settings. Each simulated dataset consists of $n=60$ observations with the number of clusters either $K=2$ or 4 , and the number of features either $p=150$ or 500. In each setting, only the first 20 features are informative and remaining features are noninformative. The samples $X_{i}, \in \mathbb{R}^{p}, i=1, \ldots, n$, are generated as follows. Denote a $p$-dimensional multivariate normal distribution as $\mathrm{MVN}_{p}$. For each $i$, a cluster label $Z_{i}$ is uniformly sampled from $\{1, \ldots, K\}$, and then the first 20 informative features are generated from $\operatorname{MVN}_{p}\left(\boldsymbol{\mu}_{K}\left(Z_{i}\right), \mathbf{I}_{20}\right)$, where $\boldsymbol{\mu}_{K}\left(Z_{i}\right)$ is defined as follows:

- If $K=2, \boldsymbol{\mu}_{2}\left(Z_{i}\right)=\mu \mathbf{1}_{20} I\left(Z_{i}=1\right)-\mu \mathbf{1}_{20} I\left(Z_{i}=2\right)$;

- If $K=4, \boldsymbol{\mu}_{4}\left(Z_{i}\right)=\left(\mu \mathbf{1}_{10}^{\mathrm{T}},-\mu \mathbf{1}_{10}^{\mathrm{T}}\right)^{\mathrm{T}} I\left(Z_{i}=1\right)+\left(-\mu \mathbf{1}_{10}^{\mathrm{T}},-\mu \mathbf{1}_{10}^{\mathrm{T}}\right)^{\mathrm{T}} I\left(Z_{i}=2\right)+$ $\left(-\mu \mathbf{1}_{10}^{\mathrm{T}}, \mu \mathbf{1}_{10}^{\mathrm{T}}\right)^{\mathrm{T}} I\left(Z_{i}=3\right)+\left(\mu \mathbf{1}_{10}^{\mathrm{T}}, \mu \mathbf{1}_{10}^{\mathrm{T}}\right)^{\mathrm{T}} I\left(Z_{i}=4\right)$,

where $\mu$ controls the distance between cluster centers. Here a large $\mu$ indicates that clusters are well-separated, whereas a small $\mu$ indicates that clusters are overlapped. Finally, the rest $p-20$ noise features are generated from $\mathcal{N}(0,1)$.

Second, we consider a non-spherical setting with two half moons. Each simulated dataset consists of $n=100$ observations with $K=2$ clusters and $p=40$ features. Only the first 
two features are informative, and the rest 38 noisy features are generated from $\mathcal{N}(0,0.5)$. It is a relatively hard setting because the number of non-informative features are 19 times more than that of informative features. Figure 3 shows one example of two interlocking half moons with the first two features. For a comparison purpose, we also apply the spectral clustering (SPECC, see Ng et al. (2002)) via an R package "kernlab" because SPECC can internally tackle non-spherical clusters.

Figure 3: The plot of the first two features for one example of two interlocking half moons.

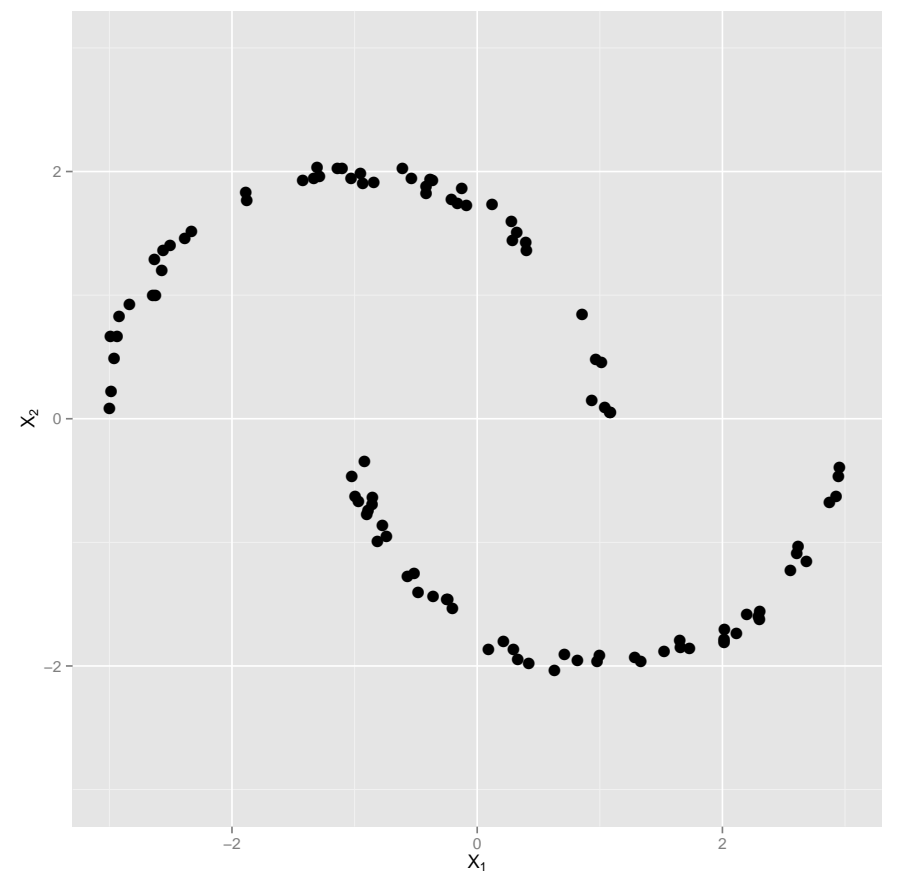

In summary, five simulation settings are considered. Setting 1: $K=2, n=60, p=150$, and $\mu=0.6$; Setting 2: $K=2, n=60, p=500$, and $\mu=0.7$; Setting $3: K=4, n=60, p=150$, and $\mu=0.9$; Setting 4: $K=4, n=60, p=500$, and $\mu=1.2$; Setting 5: two half moons with $K=2, n=100, p=40$. For each setting, we run 200 repetitions.

The RAND index (Rand, 1971) is used to measure the agreement between the estimated clustering result and the underlying true clustering assignment. The RAND index ranges between 0 and 1, and a higher value indicates better performance. Note that the true cluster labels are known in simulation studies, and thus it is feasible to know how well the candidate methods can perform if they are tuned by maximizing the RAND index. To ensure fair comparisons, for each repetition, 
separate validation samples are generated and used to select an optimal $k$ in k-means, an optimal $\gamma$ in ADMM and AMA, and optimal $\gamma_{1}$ and $\gamma_{2}$ in S-ADMM and S-AMA. To evaluate the performance of variable selection, two measurements are reported: the false negative rate (FNR) and the false positive rate (FPR). All the simulation results are summarized in Table 2 . Due to its relatively expensive computational costs, S-ADMM is not evaluated for Settings 2 and 4 where $p=500$.

In the first four simulation settings, the centers are spherical and hence k-means always performs well in clustering accuracy, i.e., large RAND index. The goals of these simulations are to justify that (1) convex clustering does not perform well when the feature dimension is high; (2) sparse convex clustering performs very well when the feature dimension is high; and (3) sparse convex clustering selects informative features with great clustering accuracy. The goal of Setting 5 is to show under the non-spherical setting, sparse convex clustering can still perform well, even better than the SPECC. All these claims are justified by the results presented in Table 2 .

First, convex clustering does not perform well when the feature dimension is high, even much worse than k-means. Similar phenomenon was also observed in the simulation studies conducted in Tan and Witten (2015). This is the motivation for developing sparse convex clustering. Second, sparse convex clustering improves convex clustering significantly. Sparse convex clustering (SAMA) performs as well as $\mathrm{k}$-means when $p=150$, and performs better than $\mathrm{k}$-means when $p=$ 500. Third, sparse convex clustering selects informative feature with great accuracy, that is, with low FNR and FPR. The feature selection performance of sparse convex clustering is very promising for settings where $p=500$. Compared with S-ADMM, S-AMA is computationally faster and also delivers slightly better accuracy. Therefore, we recommend S-AMA in practice. Fourth, for Setting 5, due to its non-spherical essential, convex clustering outperforms k-means. Surprisingly, SPECC does not outstand in terms of the RAND index. The existence of many noninformative features undermines the SPECC and it confirms the necessity of selecting informative features. Sparse convex clustering performs the best by selecting informative features. 
Table 2: Empirical mean and standard deviation (SD) of the RAND index, false positive rate (FPR), and false negative rate (FNR) based on 200 repetitions. Setting 1: $K=2, n=60, p=150$, and $\mu=0.6$; Setting 2: $K=2, n=60, p=500$, and $\mu=0.7$; Setting 3: $K=4, n=60, p=150$, and $\mu=0.9$; Setting 4: $K=4, n=60, p=500$, and $\mu=1.2$; Setting 5: two half moons with $K=2, n=100, p=40$. The best RAND index in each scenario is shown in bold.

\begin{tabular}{llllllll}
\hline & & \multicolumn{2}{c}{ RAND } & \multicolumn{2}{c}{ FNR } & \multicolumn{2}{c}{ FPR } \\
\hline & Algorithm & mean & SD & mean & SD & mean & SD \\
\hline \hline Setting 1 & k-means & 0.95 & 0.06 & 0.00 & 0.00 & 1.00 & 0.00 \\
& ADMM & 0.53 & 0.39 & 0.00 & 0.00 & 1.00 & 0.00 \\
& AMA & 0.66 & 0.40 & 0.00 & 0.00 & 1.00 & 0.00 \\
& S-ADMM & 0.82 & 0.24 & 0.04 & 0.05 & 0.25 & 0.16 \\
& S-AMA & $\mathbf{0 . 9 6}$ & 0.06 & 0.03 & 0.07 & 0.30 & 0.21 \\
\hline Setting 2 & k-means & 0.95 & 0.11 & 0.00 & 0.00 & 1.00 & 0.00 \\
& ADMM & 0.14 & 0.20 & 0.00 & 0.00 & 1.00 & 0.00 \\
& AMA & 0.08 & 0.21 & 0.00 & 0.00 & 1.00 & 0.00 \\
& S-AMA & $\mathbf{0 . 9 7}$ & 0.07 & 0.07 & 0.09 & 0.11 & 0.10 \\
\hline Setting 3 & k-means & 0.83 & 0.15 & 0.00 & 0.00 & 1.00 & 0.00 \\
& ADMM & 0.56 & 0.22 & 0.00 & 0.00 & 1.00 & 0.00 \\
& AMA & 0.47 & 0.21 & 0.00 & 0.00 & 1.00 & 0.00 \\
& S-ADMM & 0.82 & 0.14 & 0.04 & 0.06 & 0.25 & 0.24 \\
& S-AMA & $\mathbf{0 . 8 4}$ & 0.13 & 0.02 & 0.04 & 0.11 & 0.18 \\
\hline ketting 4 & k-means & 0.89 & 0.14 & 0.00 & 0.00 & 1.00 & 0.00 \\
& ADMM & 0.31 & 0.23 & 0.00 & 0.00 & 1.00 & 0.00 \\
& AMA & 0.31 & 0.20 & 0.00 & 0.00 & 1.00 & 0.00 \\
& S-AMA & $\mathbf{0 . 9 4}$ & 0.09 & 0.01 & 0.02 & 0.01 & 0.03 \\
\hline & k-means & 0.51 & 0.07 & 0.00 & 0.00 & 1.00 & 0.00 \\
& ADMM & 0.54 & 0.08 & 0.00 & 0.00 & 1.00 & 0.00 \\
& AMA & 0.53 & 0.09 & 0.00 & 0.00 & 1.00 & 0.00 \\
& S-AMA & $\mathbf{0 . 5 7}$ & 0.07 & 0.00 & 0.00 & 0.34 & 0.27 \\
& SPECC & 0.52 & 0.08 & 0.00 & 0.00 & 1.00 & 0.00 \\
\hline
\end{tabular}

\subsection{Application}

We evaluate the performance of sparse convex clustering in LIBRAS movement data from the Machine Learning Repository (Lichman, 2013). The original dataset contains 15 classes with each class referring to a hand movement type. Each class contains 24 observations, and each observation has 90 features consisting of the coordinates of hand movements. We use this dataset without the clustering assignments to evaluate each clustering algorithms and then compare the results with the true classes to compute the RAND index. Before cluster analysis, each feature is centered. In 
our S-AMA algorithm, we set $m=5$ and $\phi=1$ for weight $w_{i_{1}, i_{2}}$.

Note that some of the original 15 clusters indicate similar hand movements, such as curved/vertical swing and horizontal/vertical straight-line. By plotting the first two principal components of the 90 features, one can see that some clusters are severely overlapped. Therefore, for evaluation purpose, six clusters, including vertical swing (labeled as 3), anti-clockwise arc (labeled as 4), clockwise arc (labeled as 5), horizontal straight-line (labeled as 7), horizontal wavy (labeled as 11), and vertical wavy (labeled as 12) in the original dataset are selected. The left panel of Figure 4 displays the plot of the first principal component (PC1) against the second principal component (PC2) of 90 features for the selected six clusters with the true cluster labels.

Figure 4: Left panel shows the plot of the first principal component against the second principal component of 90 features for the selected six clusters with true cluster labels; Right panel shows the clustering path of convex clustering (AMA) using all 90 features by plotting the first principal component (PC1) against the second principal component (PC2).
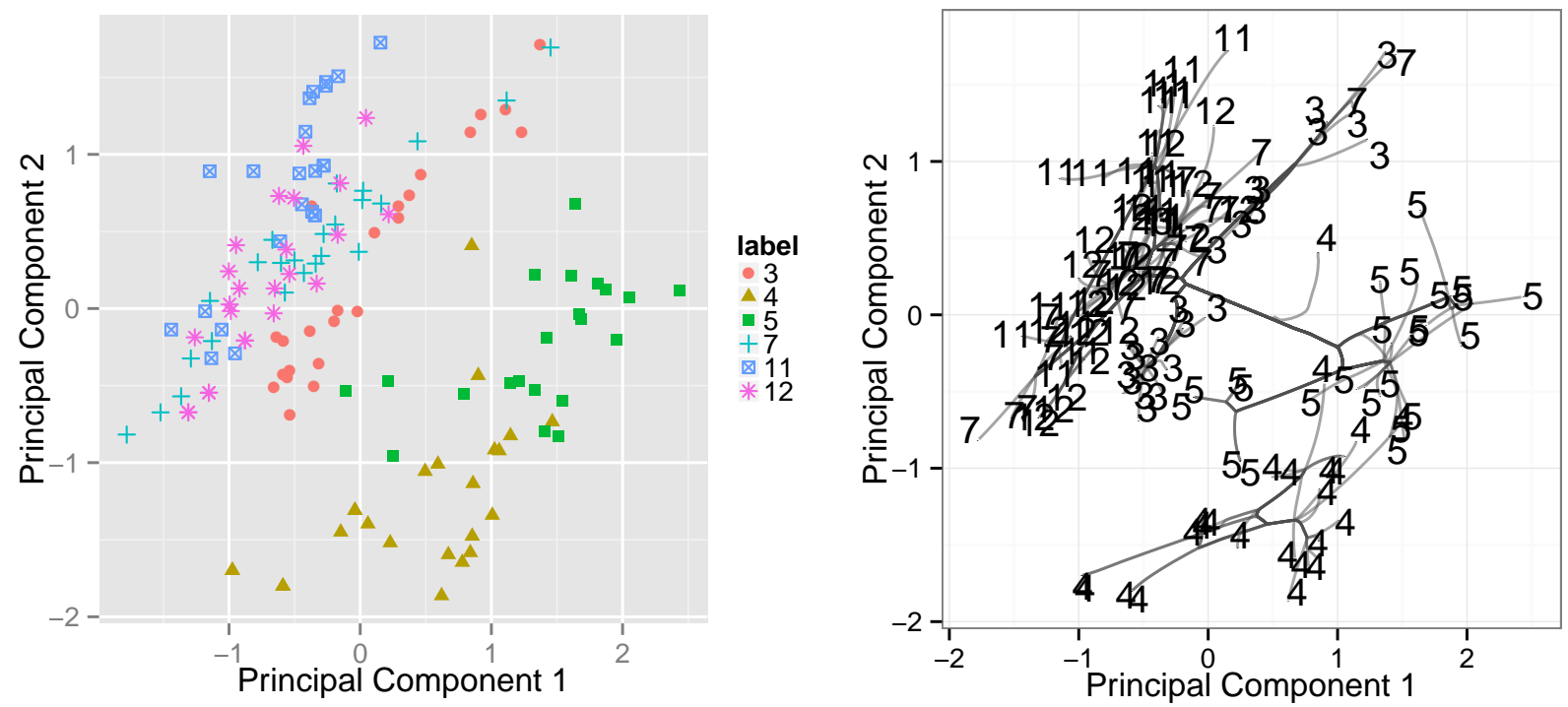

We first display the clustering path of convex clustering (AMA) using all 90 features in the right panel of Figure 4. Clearly, convex clustering is only able to distinguish clusters 4 and 5 and treat the rest clusters as one class. This phenomenon shows the curse of dimensionality in highdimensional clustering and motivates the need to conduct feature selection for improved clustering performance. 
We use S-AMA to solve sparse convex clustering. The tuning parameters are selected according to the stability selection in Section 4.2. Table 3 reports the number of estimated clusters, the number of selected features, and the RAND index between the estimated cluster membership and the true cluster membership for k-means, AMA algorithm, and our S-AMA algorithm. Clearly, both convex clustering (AMA) and sparse convex clustering (S-AMA) perform better than k-means, which indicates that the performance of convex clustering or sparse convex clustering is less sensitive to the assumption of spherical clustering centers. In addition, by using only 13 informative features, our S-AMA is able to improve the clustering accuracy of convex clustering (AMA) by $45 \%$. This indicates the importance of variable selection in high-dimensional clustering.

Table 3: The number of estimated clusters, the number of selected features, and the RAND index for k-means, AMA, and our S-AMA algorithm.

\begin{tabular}{c|ccc}
\hline Algorithm & \# of clusters & \# of features & RAND index \\
\hline k-means & 2 & 90 & 0.06 \\
AMA & 3 & 90 & 0.31 \\
S-AMA & 3 & 13 & $\mathbf{0 . 4 5}$ \\
\hline
\end{tabular}

Next we demonstrate the clustering path of sparse convex clustering (S-AMA) with the 13 selected features in Figure 5. Figure 5 displays three big clusters, which is consistent with the number of estimated clusters shown in Table 3. As tuning parameter $\gamma_{1}$ increases, the clustering path of S-AMA tends to merge clusters 3, 7 and 12 into one big cluster, merge cluster 4 and 5 into another big cluster, and identify cluster 11 as the third cluster. This finding is displayed in the final clustering path of S-AMA executed at the selected $\gamma_{1}$ and $\gamma_{2}$ as shown in Figure 6, In the plot, the left-panel graph shows the true cluster labels and the right-panel graph shows the three estimated clusters using S-AMA.

\section{Summary}

In this paper, an extension of convex clustering, sparse convex clustering, is proposed to simultaneously cluster observations and conduct feature selection. Two algorithms, S-ADMM and S-AMA, 
Figure 5: The clustering path of sparse convex clustering (S-AMA) using only 13 selected features by plotting PC1 against PC2. These 13 features are selected via stability selection.

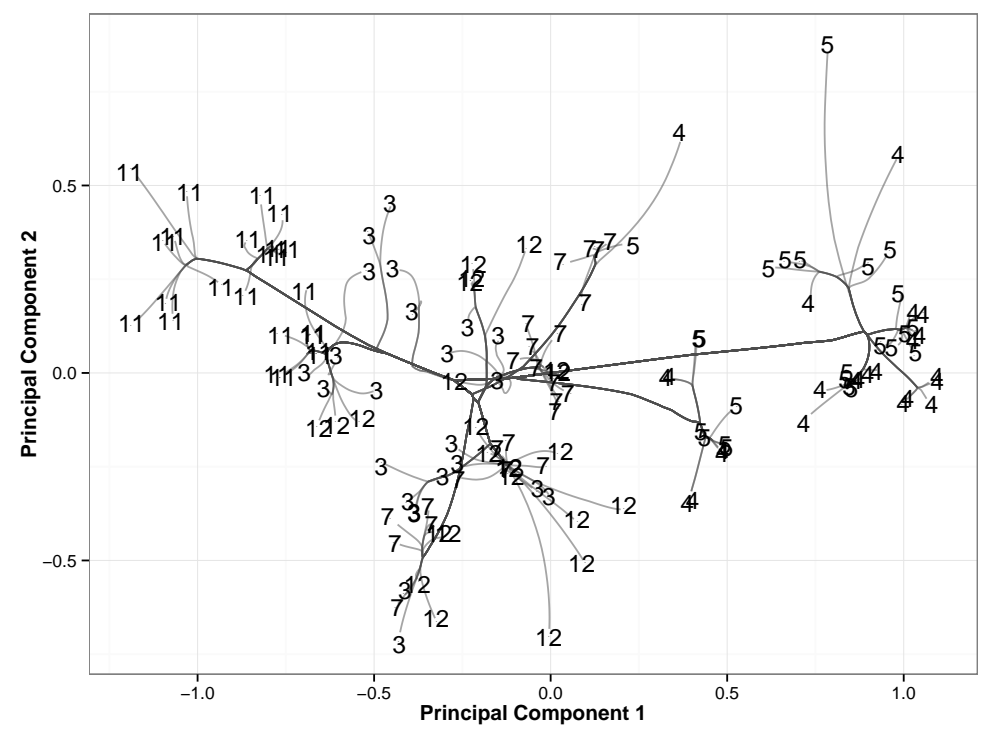

Figure 6: The left-panel graph shows the true cluster labels by plotting PC1 against PC2 using only 13 selected features. The right-panel graph shows the estimated cluster membership using S-AMA at the selected tuning parameters.

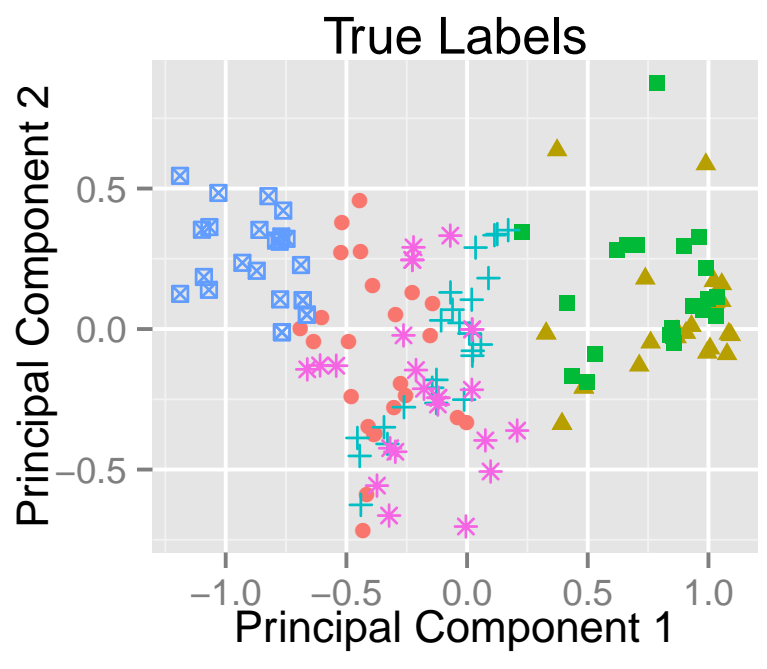

- $3 \triangle 4=5+7 \otimes 11 * 12$

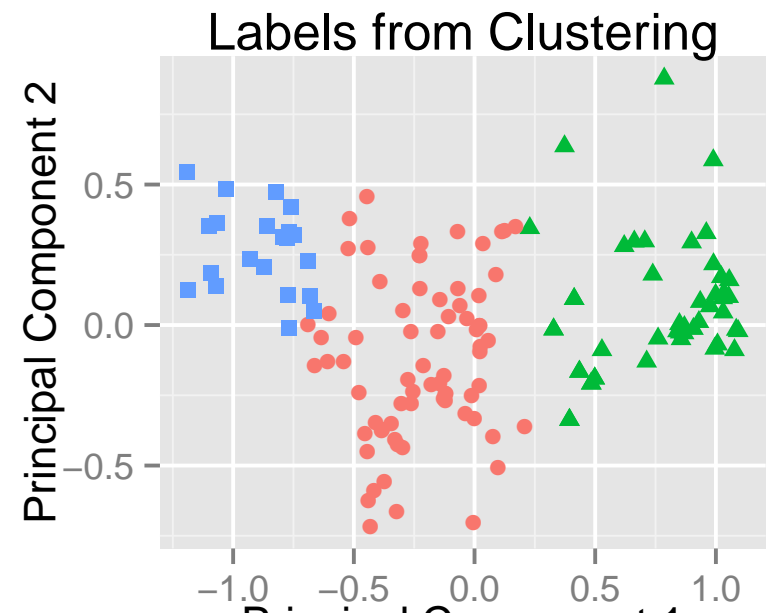

Principal Component 1

$-A \triangle B=C$

are developed to implement the new method. The numerical results show that S-AMA is computationally faster and delivers better performance than S-ADMM. In addition, the numerical results show that the selection of tuning parameters in sparse convex clustering is important and the tuning method based on clustering stability performs well. Moreover, this work can motivate future 
work. Chi et al. (2016) presented a convex formulation of the biclustering problem, which seeks to cluster observations and features at the same time. Similarly, we can extend convex biclustering to sparse convex biclustering, in order to conduct biclustering and feature selection simultaneously.

\section{References}

Alelyani, S., Tang, J., and Liu, H. (2013). Feature slection for clustering: review. In In Data Clustering: Algorithms and Applications. Edited by: Charu A, Chandan R. CRC Press.

Boyd, S., Parikh, N., Chu, E., Peleato, B., and Eckstein, J. (2011). Distributed optimization and

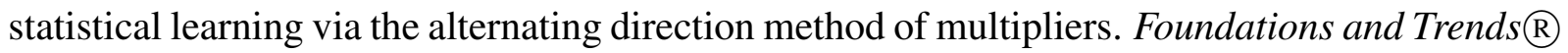
in Machine Learning, 3(1):1-122.

Chi, E. C., Allen, G. I., and Baraniuk, R. G. (2016). Convex biclustering. Biometrics.

Chi, E. C. and Lange, K. (2015). Splitting Methods for Convex Clustering. Journal of Computational and Graphical Statistics, 24(4):994-1013.

Fang, Y. and Wang, J. (2012). Selection of the number of clusters via the bootstrap method. Computational Statistics \& Data Analysis, 56:468-477.

Friedman, J., Hastie, T., and Tibshirani, R. (2010). A note on the group lasso and a sparse group lasso arXiv : 1001 . 0736v1 [ math. ST ] 5 Jan 2010. pages 1-8.

Gabay, D. and Mercier, B. (1976). A dual algorithm for the solution of nonlinear variational problems via finite element approximation. Computers \& Mathematics with Applications, 2(1):1740.

Glowinski, R. and Marroco, A. (1975). Sur l'approximation, par éléments finis d'ordre un, et la résolution, par pénalisation-dualité d'une classe de problèmes de dirichlet non linéaires. Revue française d'automatique, informatique, recherche opérationnelle. Analyse numérique, 9(2):4176. 
Guo, J., Levina, E., Michailidis, G., and Zhu, J. (2010). Pairwise variable selection for highdimensional model-based clustering. Biometrics, 66:793-804.

Hanson, D. L. and Wright, F. T. (1971). A bound on tail probabilities for quadratic forms in independent random variables. The Annals of Mathematical Statistics, 42:1079-1083.

Hocking, T. D., Joulin, A., Bach, F., and Vert, J.-P. (2011). Clusterpath : An Algorithm for Clustering using Convex Fusion Penalties. Proceedings of the 28th International Conference on Machine Learning (ICML).

Lichman, M. (2013). UCI machine learning repository. http://archive.ics.uci.edu/ ml.

Lindsten, F., Ohlsson, H., and Ljung, L. (2011). Clustering sum-of-norms regularization: With application to particle filter output computation. In Statistical Signal Processing Workshop (SSP), pages 201-204. IEEE.

Liu, J., Yuan, L., and Ye, J. (2013). Guaranteed sparse recovery under linear transformation. In Proceedings of the 30th International Conference on Machine Learning (ICML-13), pages 9199.

Ng, A. Y., Jordan, M. I., Weiss, Y., et al. (2002). On spectral clustering: Analysis and an algorithm. Advances in neural information processing systems, 2:849-856.

Pan, W. and Shen, X. (2007). Penalized model-based clustering with application to variable selection. Journal of Machine Learning Research, 8:1145-1164.

Pelckmans, K., De Brabanter, J., Suykens, J., and De Moor, B. (2005). Convex clutering shrinkage. In PASCAL Workshop on Statistics and Optimization of Clustering Workshop.

Raftery, A. and Dean, N. (2006). Variable selection for model-based clustering. Journal of the American Statistical Association, 101:168-178. 
Rand, W. M. (1971). Objective Criteria for the Evaluation of Clustering Methods. Journal of the American Statistical Association, 66(336):846-850.

Rigollet, P. (2015). 18. s997: High dimensional statistics. http://www-math.mit.edu/ rigollet/PDFs/RigNotes15.pdf.

Sun, W., Wang, J., and Fang, Y. (2012). Regularized k-means clustering of high-dimensional data and its asymptotic consistency. Electronic Journal of Statistics, 6(April 2011):148-167.

Tan, K. M. and Witten, D. (2015). Statistical properties of convex clustering. Electronic Journal of Statistics, 9:2324-2347.

Tibshirani, R. (1996). Regression shrinkage and selection via the lasso. Journal of the Royal Statistical Society, Series B, 58:267-288.

Tibshirani, R., Saunders, M., Rosset, S., Zhu, J., and Knight, K. (2005). Sparsity and smoothness via the fused lasso. Journal of the Royal Statistical Society: Series B, 67:1198-1232.

Tseng, P. (1991). Applications of a splitting algorithm to decomposition in convex programming and variational inequalities. SIAM Journal on Control and Optimization, 29(1):119-138.

Vaiter, S., Deledalle, C., Peyr, G., Fadili, J. M., Vaiter, S., Deledalle, C., Peyr, G., Fadili, J. M., and Dossal, C. (2012). The degrees of freedom of the Group Lasso for a General Design. arXiv: 1212.6478.

Wang, H. and Leng, C. (2008). A note on adaptive group lasso. Computational Statistics and Data Analysis, pages 5277-5286.

Wang, J. (2010). Consistent selection of the number of clusters via crossvalidation. Biometrika, 97:893-904.

Wang, S. and Zhu, J. (2008). Variable selection for model-based high-dimensional clustering and its application to microarray data. Biometrics, 64:440-448. 
Wang, Y., Fang, Y., and Wang, J. (2013). Sparse optimal discriminant clustering. Statistics and Computing, pages 1-11.

Witten, D. and Tibshirani, R. (2010). A framework for feature selection in clustering. Journal of the American Statistical Association, 105:713-726.

Xie, B., Pan, W., and Shen, X. (2010). Variable selection in penalized model-based clustering via regularization on grouped parameters. Biometrics, 64:921-930.

Yuan, M. and Lin, Y. (2006). Model selection and estimation in regression with grouped variables. Journal of the Royal Statistical Society: Series B (Statistical Methodology), 68(1):49-67.

Zhu, C., Xu, H., Leng, C., and Yan, S. (2014). Convex Optimization Procedure for Clustering: Theoretical Revisit. Advances in Neural Information Processing Systems, (1):1-9.

Zou, H. (2006). The adaptive lasso and its oracle properties. Journal of the American Statistical Association, 101:1418-1429. 


\section{Appendix}

In Appendix, we provide proofs of Lemma 1 and Proposition 1 . All technical details for update steps of S-AMA, proofs of Theorem 1,3, and degrees of freedom are included in Supplementary.

\section{Proof of Lemma 1}

Denote $\mathbf{a}=\operatorname{vec}(\mathbf{A})$, a vectorization of the matrix A. According to the fact that $A_{i_{1}} .-A_{i_{2}} .=$ $\mathbf{A}^{\mathrm{T}}\left(\mathbf{e}_{i_{1}}-\mathbf{e}_{i_{2}}\right)$ and the property of the tensor product $\operatorname{vec}(\mathbf{R S T})=\left[\mathbf{T}^{\mathrm{T}} \otimes \mathbf{R}\right] \operatorname{vec}(\mathbf{S})$, solving the minimization of $f(\mathbf{A})$ is equivalent to minimize

$$
f(\mathbf{a})=\frac{1}{2}\|\mathbf{x}-\mathbf{a}\|_{2}^{2}+\frac{\nu}{2} \sum_{l \in \mathcal{E}}\left\|\mathbf{B}_{l} \mathbf{P a}-\widetilde{\mathbf{v}}_{l}\right\|_{2}^{2}+\gamma_{2} \sum_{j=1}^{p} u_{j}\left\|\mathbf{a}_{j}\right\|_{2},
$$

where $\mathbf{B}_{l}=\left(\mathbf{e}_{i_{1}}-\mathbf{e}_{i_{2}}\right)^{\mathrm{T}} \otimes \mathbf{I}_{p}$ and $\mathbf{P}$ is a permutation matrix such that $\operatorname{vec}\left(\mathbf{A}^{\mathrm{T}}\right)=\operatorname{Pvec}(\mathbf{A})$ $\left(\mathbf{P}^{\mathrm{T}}=\mathbf{P}^{-1}\right)$. Letting $\mathbf{B}^{\mathrm{T}}=\left(\mathbf{B}_{1}^{\mathrm{T}}, \ldots, \mathbf{B}_{|\mathcal{E}|}^{\mathrm{T}}\right), \widetilde{\mathbf{v}}^{\mathrm{T}}=\left(\widetilde{\mathbf{v}}_{1}^{\mathrm{T}}, \ldots, \widetilde{\mathbf{v}}_{|\mathcal{E}|}^{\mathrm{T}}\right)$, it becomes

$$
f(\mathbf{a})=\frac{1}{2}\|\mathbf{x}-\mathbf{a}\|_{2}^{2}+\frac{\nu}{2}\|\mathbf{B P a}-\widetilde{\mathbf{v}}\|_{2}^{2}+\gamma_{2} \sum_{j=1}^{p} u_{j}\left\|\mathbf{a}_{j}\right\|_{2}
$$

To further simplify the formulae, the following proposition is needed, with proof shown later.

Proposition 1 For a permutation matrix $\mathbf{P}$ such that vec $\left(\mathbf{A}^{\mathrm{T}}\right)=\mathbf{P} v e c(\mathbf{A})$ and any $n$-dim vector $\mathbf{d},\left[\mathbf{d}^{\mathrm{T}} \otimes \mathbf{I}_{p}\right] \mathbf{P}=\mathbf{I}_{p} \otimes \mathbf{d}^{\mathrm{T}}$.

By Proposition 1, $\mathbf{B}_{l} \mathbf{P}=\mathbf{I}_{p} \otimes\left(\mathbf{e}_{i_{1}}-\mathbf{e}_{i_{2}}\right)^{\mathrm{T}} \triangleq \mathbf{C}_{l}$. Let $\mathbf{C}^{\mathrm{T}}=\left(\mathbf{C}_{1}^{\mathrm{T}}, \ldots, \mathbf{C}_{|\mathcal{E}|}^{\mathrm{T}}\right)$, then the second term in $f(\mathbf{a})$ becomes $\frac{\nu}{2}\|\mathbf{C a}-\widetilde{\mathbf{v}}\|_{2}^{2}=\frac{\nu}{2} \sum_{j=1}^{p} \sum_{l \in \mathcal{E}}\left(\left(\mathbf{e}_{i_{1}}-\mathbf{e}_{i_{2}}\right)^{\mathrm{T}} \mathbf{a}_{j}-\widetilde{v}_{j l}\right)_{2}^{2}$.

Therefore, the objective function can be separated to $p$ sub-optimization questions:

$$
\min _{\mathbf{a}_{j}} \frac{1}{2}\left\|\mathbf{x}_{j}-\mathbf{a}_{j}\right\|_{2}^{2}+\frac{\nu}{2} \sum_{l \in \mathcal{E}}\left(\left(\mathbf{e}_{i_{1}}-\mathbf{e}_{i_{2}}\right)^{\mathrm{T}} \mathbf{a}_{j}-\widetilde{v}_{j l}\right)_{2}^{2}+\gamma_{2} u_{j}\left\|\mathbf{a}_{j}\right\|_{2}, \quad j=1, \ldots, p .
$$

By some algebra, if $\mathcal{E}$ contains all possible edges, it can be rewritten as

$$
\min _{\mathbf{a}_{j}} \frac{1}{2} \mathbf{a}_{j}^{\mathrm{T}} \mathbf{M} \mathbf{a}_{j}-\mathbf{z}_{i}^{\mathrm{T}} \mathbf{a}_{j}+\frac{1}{2} \nu \widetilde{\mathbf{v}}_{j}^{\mathrm{T}} \widetilde{\mathbf{v}}_{j}+\gamma_{2} u_{j}\left\|\mathbf{a}_{j}\right\|_{2}
$$


where $\widetilde{\mathbf{v}}_{j} .=\left(\widetilde{v}_{j 1}, \ldots, \widetilde{v}_{j|\mathcal{E}|}\right)^{\mathrm{T}}, \mathbf{M}=\mathbf{I}_{n}+\nu \sum_{l \in \mathcal{E}}\left(\mathbf{e}_{i_{1}}-\mathbf{e}_{i_{2}}\right)\left(\mathbf{e}_{i_{1}}-\mathbf{e}_{i_{2}}\right)^{\mathrm{T}}=(1+n \nu) \mathbf{I}_{n}-\nu \mathbf{1}_{n} \mathbf{1}_{n}^{\mathrm{T}}$, and $\mathbf{z}_{j}=\mathbf{x}_{j}+\nu \sum_{l \in \mathcal{E}} \widetilde{v}_{j l}\left(\mathbf{e}_{i_{1}}-\mathbf{e}_{i_{2}}\right)$. The KKT conditions of (A.1) are

$$
\forall \mathbf{a}_{j} \neq \mathbf{0}, \quad \mathbf{M} \mathbf{a}_{j}-\mathbf{z}_{i}+\frac{\gamma_{2} u_{j} \mathbf{a}_{j}}{\left\|\mathbf{a}_{j}\right\|_{2}}=\mathbf{0} ; \quad \forall \mathbf{a}_{j}=\mathbf{0}, \quad\left\|\mathbf{z}_{j}\right\|_{2} \leqslant \gamma_{2} u_{j}
$$

Here are some remarks on the above KKT conditions. $\mathbf{M}$ is positive definite, and thus it can be diagonalized by $\mathbf{M}=\mathbf{S}^{\mathrm{T}} \boldsymbol{\Phi} \mathbf{S}$, where $\boldsymbol{\Phi}=\operatorname{diag}\left(\phi_{1}, \ldots, \phi_{n}\right)$ and $\mathbf{S}$ is an orthogonal matrix. It can be verified that $\phi_{1}=1$ and $\phi_{i}=1+n \nu, i=2, \ldots, n$. Then $\mathbf{\Phi} \mathbf{S a}_{j}-\mathbf{S z}_{j}+\frac{\gamma_{2} u_{j} \mathbf{S a}_{j}}{\left\|\mathbf{S} \mathbf{a}_{j}\right\|_{2}}=\mathbf{0}$. Let $\widetilde{\mathbf{a}}_{j}=\mathbf{S} \mathbf{a}_{j}$. One needs to solve $\boldsymbol{\Phi} \widetilde{\mathbf{a}}_{j}-\mathbf{S z}_{j}+\frac{\gamma_{2} u_{j} \widetilde{\mathbf{a}}_{j}}{\left\|\widetilde{\mathbf{a}}_{j}\right\|_{2}}=\mathbf{0}$. If $\nu=0$, then $\boldsymbol{\Phi}=\mathbf{I}$, implying the solution $\widetilde{\mathbf{a}}_{j}$ shares the same direction with $\mathbf{z}_{j}$. In this case, an explicit soft-threshold formula can be obtained, and this situation under the standard group LASSO problem was discussed in Yuan and Lin (2006). But if $\nu \neq 0$, a scaling transformation is applied to $\widetilde{\mathbf{a}}_{j}$, and there is no explicit solution.

Alternatively, we can rewrite A.1 so that existing algorithms can be applied. Define $\mathbf{N}=$ $\sqrt{1+n \nu} \mathbf{I}_{n}-\frac{\sqrt{1+n \nu}-1}{n} \mathbf{1}_{n} \mathbf{1}_{n}^{\mathrm{T}}$, which performs like a "design matrix". It can be verified that $\mathbf{M}=$ $\mathbf{N N}$ and $\mathbf{N}^{-1}$ has the form defined in Lemma 1. Let $\mathbf{y}_{j}=(\mathbf{N})^{-1} \mathbf{z}_{j}$, which performs like a pseudo outcome in the $j$-th sub-problem. Then A.1 is equivalent to $\min _{\mathbf{a}_{j}} \frac{1}{2}\left\|\mathbf{y}_{j}-\mathbf{N a} \mathbf{a}_{j}\right\|_{2}^{2}+\gamma_{2} u_{j}\left\|\mathbf{a}_{j}\right\|_{2}$. Note that during the whole algorithm, $\mathbf{N}$ and its inverse are calculated only once. This ends the proof of Lemma 1 .

Proof of Proposition 1: Note that $\mathbf{P}=\left(P_{k l}\right), 1 \leqslant k, l \leqslant n p$ here is a unique permutation matrix such that $P_{k l}=1$ if $k=(i-1) p+j$ and $l=(j-1) n+i, 1 \leqslant i \leqslant n, 1 \leqslant j \leqslant p$, and 0 otherwise. By the definition of $\mathbf{P}$, it is clear that multiplying a matrix by $\mathbf{P}$ on the right moves its $k$-th column to the $l$-th column when $P_{k l}=1$.

Consider the $i$-th element $d_{i}$ of $\mathbf{d}$, then in $\mathbf{d}^{\mathrm{T}} \otimes \mathbf{I}_{p}$, its entries at $(j,(i-1) p+j)$ equal $d_{i}$, $j=1, \ldots, p$. Thus, in $\left(\mathbf{d}^{\mathrm{T}} \otimes \mathbf{I}_{n}\right) \mathbf{P}$, the entry at $(j,(j-1) n+i)$ equals to $d_{i}$. In $\mathbf{I}_{p} \otimes \mathbf{d}^{\mathrm{T}}$, it is easy to see the entry at $(j,(j-1) n+i)$ equal $d_{i}, i=1, \ldots, n, j=1, \ldots, p$. 


\title{
Supplementary Materials for: Sparse Convex Clustering
}

\author{
Binhuan Wang 1 节, Yilong Zhang ${ }^{2}$, Will Wei Sun ${ }^{3}$, Yixin Fang ${ }^{4}$ \\ ${ }^{1}$ New York University School of Medicine \\ ${ }^{2}$ Merck Research Laboratories \\ ${ }^{3}$ University of Miami School of Business Administration \\ ${ }^{4}$ Department of Mathematical Sciences, New Jersey Institute of Technology
}

In this supplementary, we provide all technical details for update steps of S-AMA, proofs of Theorems 1,3 and degrees of freedom.

\section{S.1 Update Steps of S-AMA}

By letting $\nu=0$ while updating $\mathbf{A}$, the S-ADMM algorithm can be simplified significantly. Noting that $\mathbf{M}=\mathbf{I}_{n}$ and $\mathbf{z}_{j}=\mathbf{x}_{j}+\sum_{l \in \mathcal{E}} \lambda_{j l}\left(\mathbf{e}_{i_{1}}-\mathbf{e}_{i_{2}}\right)$, where $\lambda_{j l}$ is the $j$-th element of $\boldsymbol{\lambda}_{l}$, the KKT conditions are

$$
\forall \mathbf{a}_{j} \neq \mathbf{0}, \quad \mathbf{a}_{j}-\mathbf{z}_{j}+\frac{\gamma_{2} u_{j} \mathbf{a}_{j}}{\left\|\mathbf{a}_{j}\right\|}=\mathbf{0} ; \quad \forall \mathbf{a}_{j}=\mathbf{0}, \quad\left\|\mathbf{z}_{i}\right\|_{2} \leqslant \gamma_{2} u_{j}
$$

The solutions are $\widehat{\mathbf{a}}_{j}=\left(1-\frac{\gamma_{2} u_{j}}{\left\|\mathbf{z}_{j}\right\|_{2}}\right)_{+} \mathbf{z}_{j}$, where $(z)_{+}=\max \{0, z\}$. See Yuan and Lin (2006).

By applying the projection method, one can update $\mathbf{v}_{l}$ and $\boldsymbol{\lambda}_{l}$ as $\mathbf{v}_{l}^{m+1}=A_{i_{1}}^{m+1}-A_{i_{2}}^{m+1}$. $\nu^{-1} \boldsymbol{\lambda}_{l}^{m}-\mathcal{P}_{t B}\left[A_{i_{1}}^{m+1}-A_{i_{2}}^{m+1}-\nu^{-1} \boldsymbol{\lambda}_{l}^{m}\right]$, where $t=\sigma_{l}=\gamma_{1} w_{l} / \nu$ and $\mathcal{P}_{B}(\mathbf{z})$ denotes projection onto $B=\left\{\mathbf{y}:\|\mathbf{y}\|_{\dagger} \leqslant 1\right\}$. The point $\mathcal{P}_{B}(\mathbf{z})$ can be characterized by the relations $\mathcal{P}_{B}(\mathbf{z}) \in B$ and $\forall \mathbf{y} \in B,\left\langle\mathbf{y}-\mathcal{P}_{B}(\mathbf{z}), \mathbf{z}-\mathcal{P}_{B}(\mathbf{z})\right\rangle \leqslant 0$. Then

$$
\begin{aligned}
\boldsymbol{\lambda}_{l}^{m+1} & =\boldsymbol{\lambda}_{l}^{m}+\nu\left(\mathbf{v}_{l}^{m+1}-A_{i_{1} \cdot}^{m+1}+A_{i_{2} \cdot}^{m+1}\right) \\
& =-\nu \mathcal{P}_{t B}\left(A_{i_{1}}^{m+1}-A_{i_{2} \cdot}^{m+1}-\nu^{-1} \boldsymbol{\lambda}_{1 l}^{m}\right) \\
& =\mathcal{P}_{C_{l}}\left(\boldsymbol{\lambda}_{l}^{m}-\nu \mathbf{g}_{l}^{m+1}\right),
\end{aligned}
$$

where $\mathbf{g}_{l}^{m}=A_{i_{1}}^{m}$. $-A_{i_{2}}^{m}$. and $C_{l}=\left\{\boldsymbol{\lambda}_{l}:\left\|\boldsymbol{\lambda}_{l}\right\|_{\dagger} \leqslant \gamma_{1} w_{l}\right\}$. Note that there is no need to update $\mathbf{v}_{l}$, and $\lambda_{l}$ can be directly updated.

\section{S.2 Proofs of Theorems $1-3$}

Before we prove the main theorems, we introduce the following lemma to bound the quadratic forms of independent sub-Gaussian random variables. This is a standard result in Hanson and Wright (1971).

\footnotetext{
${ }^{\dagger}$ Correspondence to: 650 First Avenue Rm 578, New York, NY 10016; Email: Binhuan.Wang @nyumc.org
} 
Lemma S.1 Let $\epsilon$ be a d-dim vector of independent sub-Gaussian random variables with mean zero and variance $\sigma^{2}$. Let $\mathbf{A}$ be a symmetric matrix. For any $t>0$, there exists some positive constants $c_{1}, c_{2}$ such that,

$$
P\left(\boldsymbol{\epsilon}^{\top} \mathbf{A} \boldsymbol{\epsilon}>\mathbf{t}+\sigma^{2} \operatorname{tr}(\mathbf{A})\right) \leqslant \exp \left\{-\min \left(\frac{c_{1} t^{2}}{\sigma^{2}\|\mathbf{A}\|_{F}}, \frac{c_{2} t}{\sigma^{2}\|\mathbf{A}\|_{s p}}\right)\right\}
$$

In addition, we need the following lemma for tail probability of a linear combination of independent sub-Gaussian random variables. This is a standard result in Rigollet (2015).

Lemma S.2 Let $\epsilon$ be a d-dim vector of independent sub-Gaussian random variables with mean zero and variance $\sigma^{2}$. Then for any $\mathbf{b} \in \mathbb{R}^{d}$, we have

$$
P\left(\mathbf{b}^{\mathrm{T}} \boldsymbol{\epsilon}>t\right) \leqslant \exp \left(-\frac{t^{2}}{2 \sigma^{2}\|\mathbf{b}\|_{2}^{2}}\right) \quad \text { and } \quad P\left(\mathbf{b}^{\mathrm{T}} \boldsymbol{\epsilon}<-t\right) \leqslant \exp \left(-\frac{t^{2}}{2 \sigma^{2}\|\mathbf{b}\|_{2}^{2}}\right)
$$

We apply the matrix decomposition proposed by Liu et al. (2013) to simplify the analysis. According to Lemma 1 in Tan and Witten (2015), the rank of $\mathbf{C}$ is $p(n-1)$. Let $\mathbf{C}=\mathbf{R D S}_{\psi}^{\mathrm{T}}$

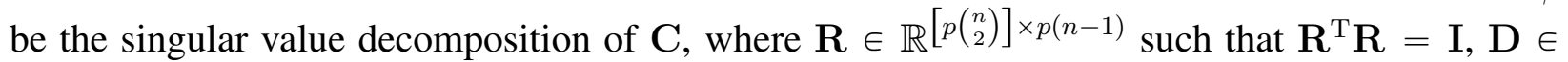
$\mathbb{R}^{p(n-1) \times p(n-1)}$ is a diagonal matrix, and $\mathbf{S}_{\psi} \in \mathbb{R}^{n p \times p(n-1)}$ such that $\mathbf{S}_{\psi}^{\mathrm{T}} \mathbf{S}_{\psi}=\mathbf{I}$. There must exist a matrix $\mathbf{S}_{\phi} \in \mathbb{R}^{n p \times p}$ such that $\mathbf{S}=\left[\mathbf{S}_{\phi}, \mathbf{S}_{\psi}\right] \in \mathbb{R}^{n p \times n p}$ is an orthogonal matrix. Then it is clear that $\mathbf{S}_{\phi}^{\mathrm{T}} \mathbf{S}_{\psi}=\mathbf{0}$.

Additionally, let $\boldsymbol{\phi}=\mathbf{S}_{\phi}^{\mathrm{T}} \mathbf{a} \in \mathbb{R}^{p}$ and $\boldsymbol{\psi}=\mathbf{S}_{\psi}^{\mathrm{T}} \mathbf{a} \in \mathbb{R}^{p(n-1)}$, and thus $\mathbf{a}=\mathbf{S}_{\phi} \boldsymbol{\phi}+\mathbf{S}_{\psi} \boldsymbol{\psi}$. Similarly, we define $\phi_{0}=\mathbf{S}_{\phi}^{\mathrm{T}} \mathbf{a}_{0}$ and $\boldsymbol{\psi}_{0}=\mathbf{S}_{\psi}^{\mathrm{T}} \mathbf{a}_{0}$, and thus $\mathbf{a}_{0}=\mathbf{S}_{\phi} \boldsymbol{\phi}_{0}+\mathbf{S}_{\psi} \boldsymbol{\psi}_{0}$.

Therefore, we can rewrite the above minimization problem as follows:

$$
\min _{\boldsymbol{\phi}, \boldsymbol{\psi}} \frac{1}{2}\left\|\mathbf{x}-\mathbf{S}_{\phi} \boldsymbol{\phi}-\mathbf{S}_{\psi} \boldsymbol{\psi}\right\|_{2}^{2}+\gamma_{1} \sum_{l \in \mathcal{E}}\left\|\mathbf{G}_{l} \boldsymbol{\psi}\right\|_{q}+\gamma_{2} \sum_{j=1}^{p} u_{j}\left\|\mathbf{E}_{j}\left(\mathbf{S}_{\phi} \boldsymbol{\phi}+\mathbf{S}_{\psi} \boldsymbol{\psi}\right)\right\|_{2}
$$

where $\mathbf{E}_{j}=\mathbf{e}_{j}^{* T} \otimes \mathbf{I}_{n}$ and $\mathbf{G}_{l}$ is a submatrix of $\mathbf{G}=\left(\mathbf{G}_{1}^{\mathrm{T}}, \ldots, \mathbf{G}_{|\mathcal{E}|}^{\mathrm{T}}\right)^{\mathrm{T}}$ such that $\mathbf{G}=\mathbf{R D}$. Note that the rank of $\mathbf{G}$ is $p(n-1)$. Thus there exists the Moore-Penrose pseudo-inverse $\mathrm{G}^{+} \in$ $\mathbb{R}^{p(n-1) \times\left[p \cdot\left(\begin{array}{l}n \\ 2\end{array}\right)\right]}$ such that $\mathbf{G}^{+} \mathbf{G}=\mathbf{I}$. Let $\widehat{\boldsymbol{\phi}}$ and $\widehat{\boldsymbol{\psi}}$ are the solution to $(\mathbf{S} .1)$. Then it is natural to see $\widehat{\boldsymbol{\phi}}=\mathbf{S}_{\phi}^{\mathrm{T}} \widehat{\mathbf{a}}, \widehat{\boldsymbol{\psi}}=\mathbf{S}_{\psi}^{\mathrm{T}} \widehat{\mathbf{a}}$ and thus $\widehat{\mathbf{a}}=\mathbf{S}_{\phi} \widehat{\boldsymbol{\phi}}+\mathbf{S}_{\psi} \widehat{\boldsymbol{\psi}}$.

Proof of Theorem 1: $\widehat{\phi}$ and $\widehat{\psi}$ are the minimizers to (S.1), so we know

$$
\begin{aligned}
& \frac{1}{2}\left\|\mathbf{x}-\mathbf{S}_{\phi} \widehat{\boldsymbol{\phi}}-\mathbf{S}_{\psi} \widehat{\boldsymbol{\psi}}\right\|_{2}^{2}+\gamma_{1}\|\mathbf{G} \widehat{\boldsymbol{\psi}}\|_{1}+\gamma_{2} \sum_{j=1}^{p} u_{j}\left\|\mathbf{E}_{j}\left(\mathbf{S}_{\phi} \widehat{\boldsymbol{\phi}}+\mathbf{S}_{\psi} \widehat{\boldsymbol{\psi}}\right)\right\|_{2} \\
& \leqslant \frac{1}{2}\left\|\mathbf{x}-\mathbf{S}_{\phi} \boldsymbol{\phi}_{0}-\mathbf{S}_{\psi} \boldsymbol{\psi}_{0}\right\|_{2}^{2}+\gamma_{1}\left\|\mathbf{G} \boldsymbol{\psi}_{0}\right\|_{1}+\gamma_{2} \sum_{j=1}^{p} u_{j}\left\|\mathbf{E}_{j}\left(\mathbf{S}_{\phi} \boldsymbol{\phi}_{0}+\mathbf{S}_{\psi} \boldsymbol{\psi}_{0}\right)\right\|_{2}
\end{aligned}
$$


By some algebra, it follows

$$
\begin{array}{r}
\frac{1}{2}\left\|\mathbf{S}_{\phi}\left(\widehat{\boldsymbol{\phi}}-\boldsymbol{\phi}_{0}\right)+\mathbf{S}_{\psi}\left(\widehat{\boldsymbol{\psi}}-\boldsymbol{\psi}_{0}\right)\right\|_{2}^{2} \leqslant g(\widehat{\boldsymbol{\phi}}, \widehat{\boldsymbol{\psi}})+\gamma_{1}\left(\left\|\mathbf{G} \boldsymbol{\psi}_{0}\right\|_{1}-\|\mathbf{G} \widehat{\boldsymbol{\psi}}\|_{1}\right) \\
+\gamma_{2} \sum_{j=1}^{p} u_{j}\left[\left\|\mathbf{E}_{j}\left(\mathbf{S}_{\phi} \boldsymbol{\phi}_{0}+\mathbf{S}_{\psi} \boldsymbol{\psi}_{0}\right)\right\|_{2}-\left\|\mathbf{E}_{j}\left(\mathbf{S}_{\phi} \widehat{\boldsymbol{\phi}}+\mathbf{S}_{\psi} \widehat{\boldsymbol{\psi}}\right)\right\|_{2}\right]
\end{array}
$$

where $g(\widehat{\boldsymbol{\phi}}, \widehat{\boldsymbol{\psi}})=\varepsilon^{\mathrm{T}}\left[\mathbf{S}_{\phi}\left(\widehat{\boldsymbol{\phi}}-\boldsymbol{\phi}_{0}\right)+\mathbf{S}_{\psi}\left(\widehat{\boldsymbol{\psi}}-\boldsymbol{\psi}_{0}\right)\right]$. Next, we build up the relation between $\widehat{\boldsymbol{\phi}}$ and $\phi_{0}$. The optimal condition for $\hat{\phi}$ based on $(\mathrm{S} .1)$ is

$$
-\mathbf{S}_{\phi}^{\mathrm{T}}\left(\mathbf{x}-\mathbf{S}_{\phi} \widehat{\boldsymbol{\phi}}-\mathbf{S}_{\psi} \widehat{\boldsymbol{\psi}}\right)+\boldsymbol{\xi}=\mathbf{0}
$$

where $\boldsymbol{\xi}=\gamma_{2} \sum_{j=1}^{p} u_{j} \frac{\mathbf{S}_{\phi}^{\mathrm{T}} \mathbf{E}_{j}^{\mathrm{T}} \mathbf{E}_{j}\left(\mathbf{S}_{\phi} \widehat{\boldsymbol{\phi}}+\mathbf{S}_{\psi} \widehat{\boldsymbol{\psi}}\right)}{\left\|\mathbf{E}_{j}\left(\mathbf{S}_{\phi} \widehat{\boldsymbol{\phi}}+\mathbf{S}_{\psi} \widehat{\boldsymbol{\psi}}\right)\right\|_{2}}$. Then we have $\widehat{\boldsymbol{\phi}}-\boldsymbol{\phi}_{0}=\mathbf{S}_{\phi}^{\mathrm{T}} \boldsymbol{\varepsilon}-\boldsymbol{\xi}$. Thus,

$$
\begin{aligned}
\frac{1}{n p}|g(\widehat{\boldsymbol{\phi}}, \widehat{\boldsymbol{\psi}})| & =\frac{1}{n p}\left|\boldsymbol{\varepsilon}^{\mathrm{T}}\left[\mathbf{S}_{\phi}\left(\mathbf{S}_{\phi}^{\mathrm{T}} \boldsymbol{\varepsilon}-\boldsymbol{\xi}\right)+\mathbf{S}_{\psi}\left(\widehat{\boldsymbol{\psi}}-\boldsymbol{\psi}_{0}\right)\right]\right| \\
& \leqslant \frac{1}{n p}\left|\boldsymbol{\varepsilon}^{\mathrm{T}} \mathbf{S}_{\phi} \mathbf{S}_{\phi}^{\mathrm{T}} \boldsymbol{\varepsilon}\right|+\frac{1}{n p}\left|\boldsymbol{\varepsilon}^{\mathrm{T}} \mathbf{S}_{\phi} \boldsymbol{\xi}\right|+\frac{1}{n p}\left|\varepsilon^{\mathrm{T}} \mathbf{S}_{\psi}\left(\widehat{\boldsymbol{\psi}}-\boldsymbol{\psi}_{0}\right)\right| \\
& \leqslant \frac{1}{n p}\left|\varepsilon^{\mathrm{T}} \mathbf{S}_{\phi} \mathbf{S}_{\phi}^{\mathrm{T}} \boldsymbol{\varepsilon}\right|+\frac{1}{n p}\left|\boldsymbol{\varepsilon}^{\mathrm{T}} \mathbf{S}_{\phi} \boldsymbol{\xi}\right|+\frac{1}{n p}\left\|\boldsymbol{\varepsilon}^{\mathrm{T}} \mathbf{S}_{\psi} \mathbf{G}^{+}\right\|_{\infty}\left\|\mathbf{G}\left(\widehat{\boldsymbol{\psi}}-\boldsymbol{\psi}_{0}\right)\right\|_{1}
\end{aligned}
$$

The last inequality follows from the fact $\mathbf{G}^{+} \mathbf{G}=\mathbf{I}$ and the Hölder's inequality. Now we need to establish bounds for the above three items on the right-hand side of the inequality.

Bounds for $\frac{1}{n p} \varepsilon^{\mathrm{T}} \mathbf{S}_{\phi} \mathbf{S}_{\phi}^{\mathrm{T}} \boldsymbol{\varepsilon}$ and $\left\|\varepsilon^{\mathrm{T}} \mathbf{S}_{\psi} \mathbf{G}^{+}\right\|_{\infty}$ :

Based on arguments used for Lemma 6 in Tan and Witten (2015), if follows

$$
P\left(\frac{1}{n p} \varepsilon^{\mathrm{T}} \mathbf{S}_{\phi} \mathbf{S}_{\phi}^{\mathrm{T}} \varepsilon \geqslant \sigma^{2}\left[\frac{1}{n}+\sqrt{\frac{\log (n p)}{n^{2} p}}\right]\right) \leqslant \exp \left\{-\min \left(c_{1} \log (n p), c_{2} \sqrt{p \log (n p)}\right)\right\}
$$

and

$$
P\left(\left\|\varepsilon^{\mathrm{T}} \mathbf{S}_{\psi} \mathbf{G}^{+}\right\|_{\infty} \geqslant 2 \sigma \sqrt{\frac{\log \left(p \cdot\left(\begin{array}{c}
n \\
2
\end{array}\right)\right)}{n}}\right) \leqslant \frac{2}{p \cdot\left(\begin{array}{c}
n \\
2
\end{array}\right)}
$$

Bound for $\frac{1}{n p}\left|\varepsilon^{\mathrm{T}} \mathbf{S}_{\phi} \boldsymbol{\xi}\right|$ : 
Note that $\left\|\mathbf{S}_{\phi} \boldsymbol{\xi}\right\|_{2} \leqslant\left\|\mathbf{S}_{\phi}\right\|_{2}\|\boldsymbol{\xi}\|_{2}=\|\boldsymbol{\xi}\|_{2}$ because $\left\|\mathbf{S}_{\phi}\right\|_{2}=1$. Next, we have

$$
\begin{aligned}
\|\boldsymbol{\xi}\|_{2} & =\left\|\gamma_{2} \sum_{j=1}^{p} u_{j} \frac{\mathbf{S}_{\phi}^{\mathrm{T}} \mathbf{E}_{j}^{\mathrm{T}} \mathbf{E}_{j}\left(\mathbf{S}_{\phi} \widehat{\boldsymbol{\phi}}+\mathbf{S}_{\psi} \widehat{\boldsymbol{\psi}}\right)}{\left\|\mathbf{E}_{j}\left(\mathbf{S}_{\phi} \widehat{\boldsymbol{\phi}}+\mathbf{S}_{\psi} \widehat{\boldsymbol{\psi}}\right)\right\|_{2}}\right\|_{2} \\
& \leqslant \gamma_{2} \sum_{j=1}^{p} u_{j}\left\|\mathbf{S}_{\phi}^{\mathrm{T}} \mathbf{E}_{j}^{\mathrm{T}}\right\|_{2} \\
& \leqslant \gamma_{2} \sum_{j=1}^{p} u_{j}\left\|\mathbf{E}_{j}^{\mathrm{T}}\right\|_{2} .
\end{aligned}
$$

Note the fact $\mathbf{e}_{j}^{* T} \otimes \mathbf{I}_{n}$, and then $\left\|\mathbf{E}_{j}^{\mathrm{T}}\right\|_{2}=1$. Thus, $\left\|\mathbf{S}_{\phi} \boldsymbol{\xi}\right\|_{2}^{2} \leqslant \gamma_{2}^{2}\|\mathbf{u}\|_{1}^{2}$. Based on Lemma S.2, we have

$$
P\left(\frac{1}{n p}\left|\boldsymbol{\varepsilon}^{\mathrm{T}} \mathbf{S}_{\phi} \boldsymbol{\xi}\right|>t\right) \leqslant 2 \exp \left(-\frac{n^{2} p^{2} t^{2}}{2 \sigma^{2}\left\|\mathbf{S}_{\phi} \boldsymbol{\xi}\right\|_{2}^{2}}\right) \leqslant 2 \exp \left(-\frac{n^{2} p^{2} t^{2}}{2 \sigma^{2} \gamma_{2}^{2}\|\mathbf{u}\|_{1}^{2}}\right)
$$

Setting $t=1 /(n p)$, it follows

$$
P\left(\frac{1}{n p}\left|\varepsilon^{\mathrm{T}} \mathbf{S}_{\phi} \boldsymbol{\xi}\right|>\frac{1}{n p}\right) \leqslant 2 \exp \left(-\frac{n p}{2 \sigma^{2} \gamma_{2}^{2}\|\mathbf{u}\|_{1}^{2}}\right)
$$

Therefore, by combining $(S .3)-(S .5)$ and setting $\gamma_{1}>4 \sigma \sqrt{\frac{\log \left(p \cdot\left(\begin{array}{l}n \\ 2\end{array}\right)\right)}{n}}$, we have that

$$
\frac{1}{n p} g(\widehat{\boldsymbol{\phi}}, \widehat{\boldsymbol{\psi}}) \leqslant \frac{\gamma_{1}}{2 n p}\left\|\mathbf{G}\left(\widehat{\boldsymbol{\psi}}-\boldsymbol{\psi}_{0}\right)\right\|_{1}+\sigma^{2}\left[\frac{1}{n}+\sqrt{\frac{\log (n p)}{n^{2} p}}\right]+\frac{1}{n p}
$$

holds with probability at least $1-c_{3}$.

Furthermore, it is clear that

$$
\begin{aligned}
\gamma_{2} \sum_{j=1}^{p} u_{j}\left[\left\|\mathbf{E}_{j}\left(\mathbf{S}_{\phi} \boldsymbol{\phi}_{0}+\mathbf{S}_{\psi} \boldsymbol{\psi}_{0}\right)\right\|_{2}-\left\|\mathbf{E}_{j}\left(\mathbf{S}_{\phi} \widehat{\boldsymbol{\phi}}+\mathbf{S}_{\psi} \widehat{\boldsymbol{\psi}}\right)\right\|_{2}\right] & =\gamma_{2} \sum_{j=1}^{p} u_{j}\left(\left\|\mathbf{a}_{0 j}\right\|_{2}-\left\|\widehat{\mathbf{a}}_{j}\right\|_{2}\right) \\
& \leqslant \gamma_{2} \sum_{j=1}^{p} u_{j}\left\|\mathbf{a}_{0 j}-\widehat{\mathbf{a}}_{j}\right\|_{2} \\
& \leqslant \gamma_{2}\|\mathbf{u}\|_{2}\left\|\mathbf{a}_{0}-\widehat{\mathbf{a}}\right\|_{2} \\
& \leqslant \frac{\gamma_{2}}{2}\left(\|\mathbf{u}\|_{2}^{2}+\left\|\mathbf{a}_{0}-\widehat{\mathbf{a}}\right\|_{2}^{2}\right)
\end{aligned}
$$

by noting that $\sum_{j=1}^{p}\left\|\mathbf{a}_{0 j}-\widehat{\mathbf{a}}_{j}\right\|_{2}^{2}=\left\|\mathbf{a}_{0}-\widehat{\mathbf{a}}\right\|_{2}^{2}$. Substituting the above inequality and (S.6) into (S.2), 
we obtain that

$$
\begin{aligned}
& \frac{1}{2 n p}\left\|\mathbf{S}_{\phi}\left(\widehat{\boldsymbol{\phi}}-\boldsymbol{\phi}_{0}\right)+\mathbf{S}_{\psi}\left(\widehat{\boldsymbol{\psi}}-\boldsymbol{\psi}_{0}\right)\right\|_{2}^{2} \\
\leqslant & \frac{\gamma_{1}}{2 n p}\left\|\mathbf{G}\left(\widehat{\boldsymbol{\psi}}-\boldsymbol{\psi}_{0}\right)\right\|_{1}+\sigma^{2}\left[\frac{1}{n}+\sqrt{\left.\frac{\log (n p)}{n^{2} p}\right]+\frac{1}{n^{2} p^{2}}}\right. \\
& +\frac{\gamma_{1}}{n p}\left(\left\|\mathbf{G} \boldsymbol{\psi}_{0}\right\|_{1}-\|\mathbf{G} \widehat{\boldsymbol{\psi}}\|_{1}\right)+\frac{\gamma_{2}}{2 n p}\left(\|\mathbf{u}\|_{2}^{2}+\left\|\mathbf{a}_{0}-\widehat{\mathbf{a}}\right\|_{2}^{2}\right)
\end{aligned}
$$

Therefore, it implies that

$$
\frac{1-\gamma_{2}}{2 n p}\left\|\widehat{\mathbf{a}}-\mathbf{a}_{0}\right\|_{2}^{2} \leqslant \frac{3 \gamma_{1}}{2 n p}\left\|\mathbf{C} \mathbf{a}_{0}\right\|_{1}+\frac{\gamma_{2}\|\mathbf{u}\|_{2}^{2}}{2 n p}+\sigma^{2}\left[\frac{1}{n}+\sqrt{\frac{\log (n p)}{n^{2} p}}\right]+\frac{1}{n^{2} p^{2}}
$$

holds with probability at least $1-c_{3}$.

Proof of Theorem 2; By the definition of $\widehat{\phi}$ and $\widehat{\psi}$, we know

$$
\begin{aligned}
& \frac{1}{2}\left\|\mathbf{x}-\mathbf{S}_{\phi} \widehat{\boldsymbol{\phi}}-\mathbf{S}_{\psi} \widehat{\boldsymbol{\psi}}\right\|_{2}^{2}+\gamma_{1} \sum_{l \in \mathcal{E}}\left\|\mathbf{G}_{l} \widehat{\boldsymbol{\psi}}\right\|_{2}+\gamma_{2} \sum_{j=1}^{p} u_{j}\left\|\mathbf{E}_{j}\left(\mathbf{S}_{\phi} \widehat{\boldsymbol{\phi}}+\mathbf{S}_{\psi} \widehat{\boldsymbol{\psi}}\right)\right\|_{2} \\
& \leqslant \frac{1}{2}\left\|\mathbf{x}-\mathbf{S}_{\phi} \boldsymbol{\phi}_{0}-\mathbf{S}_{\psi} \boldsymbol{\psi}_{0}\right\|_{2}^{2}+\gamma_{1} \sum_{l \in \mathcal{E}}\left\|\mathbf{G}_{l} \boldsymbol{\psi}_{0}\right\|_{2}+\gamma_{2} \sum_{j=1}^{p} u_{j}\left\|\mathbf{E}_{j}\left(\mathbf{S}_{\phi} \boldsymbol{\phi}_{0}+\mathbf{S}_{\psi} \boldsymbol{\psi}_{0}\right)\right\|_{2}
\end{aligned}
$$

implying

$$
\begin{aligned}
& \frac{1}{2}\left\|\mathbf{S}_{\phi}\left(\widehat{\boldsymbol{\phi}}-\boldsymbol{\phi}_{0}\right)+\mathbf{S}_{\psi}\left(\widehat{\boldsymbol{\psi}}-\boldsymbol{\psi}_{0}\right)\right\|_{2}^{2} \\
\leqslant & g(\widehat{\boldsymbol{\phi}}, \widehat{\boldsymbol{\psi}})+\gamma_{1} \sum_{l \in \mathcal{E}}\left(\left\|\mathbf{G}_{l} \boldsymbol{\psi}_{0}\right\|_{2}-\left\|\mathbf{G}_{l} \widehat{\boldsymbol{\psi}}\right\|_{2}\right) \\
& +\gamma_{2} \sum_{j=1}^{p} u_{j}\left[\left\|\mathbf{E}_{j}\left(\mathbf{S}_{\phi} \boldsymbol{\phi}_{0}+\mathbf{S}_{\psi} \boldsymbol{\psi}_{0}\right)\right\|_{2}-\left\|\mathbf{E}_{j}\left(\mathbf{S}_{\phi} \widehat{\boldsymbol{\phi}}+\mathbf{S}_{\psi} \widehat{\boldsymbol{\psi}}\right)\right\|_{2}\right] .
\end{aligned}
$$

Next, we build up the relation between $\widehat{\phi}$ and $\phi_{0}$. Following the same arguments used in the case with $q=1$, we have $\widehat{\phi}-\phi_{0}=\mathbf{S}_{\phi}^{\mathrm{T}} \boldsymbol{\varepsilon}-\boldsymbol{\xi}$. Thus,

$$
\begin{aligned}
\frac{1}{n p}|g(\widehat{\boldsymbol{\phi}}, \widehat{\boldsymbol{\psi}})| & =\frac{1}{n p}\left|\boldsymbol{\varepsilon}^{\mathrm{T}} \mathbf{S}_{\phi} \mathbf{S}_{\phi}^{\mathrm{T}} \boldsymbol{\varepsilon}-\boldsymbol{\varepsilon}^{\mathrm{T}} \mathbf{S}_{\phi} \boldsymbol{\xi}+\boldsymbol{\varepsilon}^{\mathrm{T}} \mathbf{S}_{\psi}\left(\widehat{\boldsymbol{\psi}}-\boldsymbol{\psi}_{0}\right)\right| \\
& \leqslant \frac{1}{n p}\left|\boldsymbol{\varepsilon}^{\mathrm{T}} \mathbf{S}_{\phi} \mathbf{S}_{\phi}^{\mathrm{T}} \boldsymbol{\varepsilon}\right|+\frac{1}{n p}\left|\boldsymbol{\varepsilon}^{\mathrm{T}} \mathbf{S}_{\phi} \boldsymbol{\xi}\right|+\frac{1}{n p}\left|\boldsymbol{\varepsilon}^{\mathrm{T}} \mathbf{S}_{\psi}\left(\widehat{\boldsymbol{\psi}}-\boldsymbol{\psi}_{0}\right)\right|
\end{aligned}
$$




$$
\begin{aligned}
& =\frac{1}{n p}\left|\boldsymbol{\varepsilon}^{\mathrm{T}} \mathbf{S}_{\phi} \mathbf{S}_{\phi}^{\mathrm{T}} \boldsymbol{\varepsilon}\right|+\frac{1}{n p}\left|\boldsymbol{\varepsilon}^{\mathrm{T}} \mathbf{S}_{\phi} \boldsymbol{\xi}\right|+\frac{1}{n p} \sum_{l \in \mathcal{E}}\left|\boldsymbol{\varepsilon}^{\mathrm{T}} \mathbf{S}_{\psi} \mathbf{G}_{l}^{+} \mathbf{G}_{l}\left(\widehat{\boldsymbol{\psi}}-\boldsymbol{\psi}_{0}\right)\right| \\
& \leqslant \frac{1}{n p}\left|\boldsymbol{\varepsilon}^{\mathrm{T}} \mathbf{S}_{\phi} \mathbf{S}_{\phi}^{\mathrm{T}} \boldsymbol{\varepsilon}\right|+\frac{1}{n p}\left|\boldsymbol{\varepsilon}^{\mathrm{T}} \mathbf{S}_{\phi} \boldsymbol{\xi}\right|+\frac{1}{n p} \sum_{l \in \mathcal{E}}\left\|\boldsymbol{\varepsilon}^{\mathrm{T}} \mathbf{S}_{\psi} \mathbf{G}_{l}^{+}\right\|_{2}\left\|\mathbf{G}_{l}\left(\widehat{\boldsymbol{\psi}}-\boldsymbol{\psi}_{0}\right)\right\|_{2} \\
& \leqslant \frac{1}{n p}\left|\boldsymbol{\varepsilon}^{\mathrm{T}} \mathbf{S}_{\phi} \mathbf{S}_{\phi}^{\mathrm{T}} \boldsymbol{\varepsilon}\right|+\frac{1}{n p}\left|\boldsymbol{\varepsilon}^{\mathrm{T}} \mathbf{S}_{\phi} \boldsymbol{\xi}\right|+\frac{1}{n p} \max _{l \in \mathcal{E}}\left\|\varepsilon^{\mathrm{T}} \mathbf{S}_{\psi} \mathbf{G}_{l}^{+}\right\|_{2} \sum_{l \in \mathcal{E}}\left\|\mathbf{G}_{l}\left(\widehat{\boldsymbol{\psi}}-\boldsymbol{\psi}_{0}\right)\right\|_{2} .
\end{aligned}
$$

Now we need to establish bounds for the above three items on the right-hand side of the inequality.

Bounds for $\frac{1}{n p} \varepsilon^{\mathrm{T}} \mathbf{S}_{\phi} \mathbf{S}_{\phi}^{\mathrm{T}} \boldsymbol{\varepsilon}$ and $\max _{l \in \mathcal{E}}\left\|\varepsilon^{\mathrm{T}} \mathbf{S}_{\psi} \mathbf{G}_{l}^{+}\right\|_{2}$ :

Based on the previous arguments used for $q=1$, if follows

$$
P\left(\frac{1}{n p} \varepsilon^{\mathrm{T}} \mathbf{S}_{\phi} \mathbf{S}_{\phi}^{\mathrm{T}} \boldsymbol{\varepsilon} \geqslant \sigma^{2}\left[\frac{1}{n}+\sqrt{\frac{\log (n p)}{n^{2} p}}\right]\right) \leqslant \exp \left\{-\min \left(c_{1} \log (n p), c_{2} \sqrt{p \log (n p)}\right)\right\}
$$

and Lemma 7 in Tan and Witten (2015) shows

$$
P\left(\max _{l \in \mathcal{E}}\left\|\varepsilon^{\mathrm{T}} \mathbf{S}_{\psi} \mathbf{G}_{l}^{+}\right\|_{2} \geqslant 2 \sigma \sqrt{\frac{\log \left(p \cdot\left(\begin{array}{c}
n \\
2
\end{array}\right)\right)}{n}}\right) \leqslant \frac{2}{p \cdot\left(\begin{array}{l}
n \\
2
\end{array}\right)}
$$

Bound for $\frac{1}{n p}\left|\varepsilon^{\mathrm{T}} \mathbf{S}_{\phi} \boldsymbol{\xi}\right|$ :

We have shown in the case with $q=1$ that

$$
P\left(\frac{1}{n p}\left|\varepsilon^{\mathrm{T}} \mathbf{S}_{\phi} \boldsymbol{\xi}\right|>\frac{1}{n p}\right) \leqslant 2 \exp \left(-\frac{n p}{2 \sigma^{2} \gamma_{2}^{2}\|\mathbf{u}\|_{1}^{2}}\right)
$$

Therefore, by combining $($ S.8 $)-\left(\right.$ S.10) and setting $\gamma_{1}>4 \sigma \sqrt{\frac{\log \left(p \cdot\left(\begin{array}{l}n \\ 2\end{array}\right)\right)}{n}}$, we have that

$$
\frac{1}{n p} g(\widehat{\boldsymbol{\phi}}, \widehat{\boldsymbol{\psi}}) \leqslant \frac{\gamma_{1}}{2 n p} \sum_{l \in \mathcal{E}}\left\|\mathbf{G}_{l}\left(\widehat{\boldsymbol{\psi}}-\boldsymbol{\psi}_{0}\right)\right\|_{2}+\sigma^{2}\left[\frac{1}{n}+\sqrt{\frac{\log (n p)}{n^{2} p}}\right]+\frac{1}{n p}
$$

holds with probability at least $1-c_{3}$.

Furthermore, we have the following results from the case with $q=1$ :

$$
\gamma_{2} \sum_{j=1}^{p} u_{j}\left[\left\|\mathbf{E}_{j}\left(\mathbf{S}_{\phi} \boldsymbol{\phi}_{0}+\mathbf{S}_{\psi} \boldsymbol{\psi}_{0}\right)\right\|_{2}-\left\|\mathbf{E}_{j}\left(\mathbf{S}_{\phi} \widehat{\boldsymbol{\phi}}+\mathbf{S}_{\psi} \widehat{\boldsymbol{\psi}}\right)\right\|_{2}\right] \leqslant \frac{\gamma_{2}}{2}\left(\|\mathbf{u}\|_{2}^{2}+\left\|\mathbf{a}_{0}-\widehat{\mathbf{a}}\right\|_{2}^{2}\right)
$$


Substituting the above inequality and $(\mathrm{S.11})$ into $(\mathrm{S.7})$, we obtain that

$$
\begin{aligned}
& \frac{1}{2 n p}\left\|\mathbf{S}_{\phi}\left(\widehat{\boldsymbol{\phi}}-\boldsymbol{\phi}_{0}\right)+\mathbf{S}_{\psi}\left(\widehat{\boldsymbol{\psi}}-\boldsymbol{\psi}_{0}\right)\right\|_{2}^{2} \\
\leqslant & \frac{\gamma_{1}}{2 n p} \sum_{l \in \mathcal{E}}\left\|\mathbf{G}_{l}\left(\widehat{\boldsymbol{\psi}}-\boldsymbol{\psi}_{0}\right)\right\|_{2}+\sigma^{2}\left[\frac{1}{n}+\sqrt{\frac{\log (n p)}{n^{2} p}}\right]+\frac{1}{n^{2} p^{2}} \\
& +\frac{\gamma_{1}}{n p} \sum_{l \in \mathcal{E}}\left(\left\|\mathbf{G}_{l} \boldsymbol{\psi}_{0}\right\|_{2}-\left\|\mathbf{G}_{l} \widehat{\boldsymbol{\psi}}\right\|_{2}\right)+\frac{\gamma_{2}}{2 n p}\left(\|\mathbf{u}\|_{2}^{2}+\left\|\mathbf{a}_{0}-\widehat{\mathbf{a}}\right\|_{2}^{2}\right) .
\end{aligned}
$$

Therefore, it implies that

$$
\frac{1-\gamma_{2}}{2 n p}\left\|\widehat{\mathbf{a}}-\mathbf{a}_{0}\right\|_{2}^{2} \leqslant \frac{3 \gamma_{1}}{2 n p} \sum_{l \in \mathcal{E}}\left\|\mathbf{C}_{l} \mathbf{a}_{0}\right\|_{2}+\frac{\gamma_{2}\|\mathbf{u}\|_{2}^{2}}{2 n p}+\sigma^{2}\left[\frac{1}{n}+\sqrt{\frac{\log (n p)}{n^{2} p}}\right]+\frac{1}{n p}
$$

holds with probability at least $1-c_{3}$.

Proof of Theorem 3;

We only need to prove $P\left(\left\|\widehat{\mathbf{a}}_{p}\right\|_{2}=0\right) \rightarrow 1$ by contradiction, the similar arguments apply to $P\left(\left\|\widehat{\mathbf{a}}_{j}\right\|_{2}=0\right) \rightarrow 1, j=p_{0}+1, \ldots, p-1$.

Case 1: $q=1$

If $\widehat{\mathbf{a}}_{p} \neq \mathbf{0}$, then $\left\|\widehat{\mathbf{a}}_{p}\right\|_{2}$ is differentiable with respect to its components. The KKT condition for $\mathbf{a}_{p}$ implies

$$
-\left(\mathbf{x}_{p}-\widehat{\mathbf{a}}_{p}\right)+\gamma_{1} \sum_{l \in \mathcal{E}} \operatorname{sgn}\left(\left(\mathbf{e}_{i_{1}}-\mathbf{e}_{i_{2}}\right)^{\mathrm{T}} \widehat{\mathbf{a}}_{p}\right)\left(\mathbf{e}_{i_{1}}-\mathbf{e}_{i_{2}}\right)+\gamma_{2} u_{p} \frac{\widehat{\mathbf{a}}_{p}}{\left\|\widehat{\mathbf{a}}_{p}\right\|_{2}}=\mathbf{0}
$$

Then it follows

$$
\frac{1}{\sqrt{n}}\left(\widehat{\mathbf{a}}_{p}-\mathbf{a}_{0 p}\right)-\frac{1}{\sqrt{n}} \boldsymbol{\varepsilon}_{p}+\frac{\gamma_{1}}{\sqrt{n}} \sum_{l \in \mathcal{E}} \operatorname{sgn}\left(\left(\mathbf{e}_{i_{1}}-\mathbf{e}_{i_{2}}\right)^{\mathrm{T}} \widehat{\mathbf{a}}_{p}\right)\left(\mathbf{e}_{i_{1}}-\mathbf{e}_{i_{2}}\right)+\frac{\gamma_{2} u_{p}}{\sqrt{n}} \frac{\widehat{\mathbf{a}}_{p}}{\left\|\widehat{\mathbf{a}}_{p}\right\|_{2}}=\mathbf{0} .
$$

Under the conditions for $\gamma_{1}$ and $\gamma_{2}$, Theorem 1 and remarks thereafter, $\mathbf{a}$ is prediction consistent, and thus $\frac{1}{n p} \sum_{j=1}^{p}\left\|\hat{\mathbf{a}}_{j}-\mathbf{a}_{0 j}\right\|_{2}^{2}=o_{P}(1)$. It implies with probability to 1 , each $\frac{1}{n}\left\|\hat{\mathbf{a}}_{j}-\mathbf{a}_{0 j}\right\|_{2}^{2}=$ $o_{P}(1)$. Then the first term is of the order $o_{P}(1)$. The second term is of the order $o_{P}(1)$ because $\varepsilon_{p}$ follows a sub-Gaussian distribution. $\left\|\gamma_{2} u_{p} \widehat{\mathbf{a}}_{p}\right\|_{2} /\left(\sqrt{n}\left\|\widehat{\mathbf{a}}_{p}\right\|_{2}\right)=\gamma_{2} u_{p} / \sqrt{n} \rightarrow 0$, and hence the forth term is of the order $o_{P}(1)$. Without loss of the generality, we assume the first entry of $\widehat{\mathbf{a}}_{p}$ is nonezero, i.e., $\widehat{A}_{1 p} \neq 0$. Also we know $\#\left\{i \mid \widehat{A}_{i p} \neq \widehat{A}_{1 p}, i=1, \ldots, n\right\} / n$ is bounded away from 0 . Thus, the first entry of $\sum_{l \in \mathcal{E}} \operatorname{sgn}\left(\left(\mathbf{e}_{i_{1}}-\mathbf{e}_{i_{2}}\right)^{\mathrm{T}} \widehat{\mathbf{a}}_{p}\right)\left(\mathbf{e}_{i_{1}}-\mathbf{e}_{i_{2}}\right)$ is of the order $O_{P}(n)$. Based on the condition $\gamma_{1}>4 \sigma \sqrt{\frac{\log \left(p \cdot\left(\begin{array}{l}n \\ 2\end{array}\right)\right)}{n}}$, we know the third term diverges to infinity and dominates other three items, which leads to a contradiction of the KKT condition. Therefore, $\widehat{\mathbf{a}}_{p}=\mathbf{0}$ with a probability tending to one. 
Case 2: $q=2$

If $\widehat{\mathbf{a}}_{p} \neq \mathbf{0}$, then $\left\|\widehat{\mathbf{a}}_{p}\right\|_{2}$ is differentiable with respect to its components. The KKT condition for $\mathbf{a}_{p}$ implies

$$
-\left(\mathbf{x}_{p}-\widehat{\mathbf{a}}_{p}\right)+\gamma_{1} \sum_{l \in \mathcal{E}} \frac{\mathbf{e}_{i_{1}}-\mathbf{e}_{i_{2}}}{\left\|\left(\mathbf{I}_{p} \otimes\left(\mathbf{e}_{i_{1}}-\mathbf{e}_{i_{2}}\right)\right) \hat{\mathbf{a}}\right\|_{2}}+\gamma_{2} u_{p} \frac{\widehat{\mathbf{a}}_{p}}{\left\|\widehat{\mathbf{a}}_{p}\right\|_{2}}=\mathbf{0}
$$

Then it follows

$$
\frac{1}{\sqrt{n}}\left(\hat{\mathbf{a}}_{p}-\mathbf{a}_{0 p}\right)-\frac{1}{\sqrt{n}} \varepsilon_{p}+\frac{\gamma_{1}}{\sqrt{n}} \sum_{l \in \mathcal{E}} \frac{\mathbf{e}_{i_{1}}-\mathbf{e}_{i_{2}}}{\left\|\left(\mathbf{I}_{p} \otimes\left(\mathbf{e}_{i_{1}}-\mathbf{e}_{i_{2}}\right)\right) \hat{\mathbf{a}}\right\|_{2}}+\frac{\gamma_{2} u_{p}}{\sqrt{n}} \frac{\widehat{\mathbf{a}}_{p}}{\left\|\widehat{\mathbf{a}}_{p}\right\|_{2}}=\mathbf{0} .
$$

Under the conditions for $\gamma_{1}$ and $\gamma_{2}$, Theorem 2 and remarks thereafter, a is prediction consistent. Similar with the arguments with the case when $q=1$ above, the first term is of the order $o_{P}(1)$. The second term is of the order $o_{P}(1)$ because $\varepsilon_{p}$ follows a sub-Gaussian distribution. $\left\|\gamma_{2} u_{p} \widehat{\mathbf{a}}_{p}\right\|_{2} /\left(\sqrt{n}\left\|\widehat{\mathbf{a}}_{p}\right\|_{2}\right)=\gamma_{2} u_{p} / \sqrt{n} \rightarrow 0$, and hence the forth term is of the order $o_{P}(1)$.

Note that all entries of $\hat{\mathbf{a}}$ cannot the same, and hence $0<\left\|\left(\mathbf{I}_{p} \otimes\left(\mathbf{e}_{i_{1}}-\mathbf{e}_{i_{2}}\right)\right) \hat{\mathbf{a}}\right\|_{2} \leqslant \|\left(\mathbf{I}_{p} \otimes\right.$ $\left.\left(\mathbf{e}_{i_{1}}-\mathbf{e}_{i_{2}}\right)\right)\left\|_{2}\right\| \widehat{\mathbf{a}}\left\|_{2} \leqslant\right\|\left(\mathbf{I}_{p} \otimes\left(\mathbf{e}_{i_{1}}-\mathbf{e}_{i_{2}}\right)\right)\left\|_{F}\right\| \hat{\mathbf{a}}\left\|_{2} \leqslant \sqrt{2 p}\right\| \hat{\mathbf{a}} \|_{2}$. Also, we have $1 /\|\widehat{\mathbf{a}}\|_{2}=O_{P}(\sqrt{n p})$. Thus, the first entry of $\sum_{l \in \mathcal{E}} \frac{\mathbf{e}_{i_{1}}-\mathbf{e}_{i_{2}}}{\left\|\left(\mathbf{I}_{p} \otimes\left(\mathbf{e}_{i_{1}}-\mathbf{e}_{i_{2}}\right)\right) \hat{\mathbf{a}}\right\|_{2}}$ is of the order $O_{P}\left(n^{\frac{3}{2}}\right)$. Based on the condition $\gamma_{1}>4 \sigma \sqrt{\frac{\log \left(p \cdot\left(\begin{array}{l}n \\ 2\end{array}\right)\right)}{n}}$, we know the third term diverges to infinity and dominates other three items, which leads to a contradiction of the KKT condition. Therefore, $\widehat{\mathbf{a}}_{p}=\mathbf{0}$ with a probability tending to one.

\section{S.3 Degrees of freedom}

In this section, we provide unbiased estimators for the degrees of freedoms of sparse convex clustering. Degrees of freedom is generally defined in regression problems to explain the amount of flexibility in the model. It is a key component for model selection and statistical hypothesis testing. Note that our sparse convex clustering can be formulated as a penalized regression problem for which the degrees of freedom can be established. Motivated by Tan and Witten (2015), we develop unbiased estimators for the degrees of freedom of sparse convex clustering with $q=1$ in Lemma $\mathbf{S . 3}$ and $q=2$ in Lemma $\mathbf{S . 4}$. For simplicity, we consider the case with $w_{l}=1, l \in \mathcal{E}$ in the following theoretical developments.

Lemma S.3 Assume $X_{i} \stackrel{\text { iid }}{\sim} M V N_{p}\left(\boldsymbol{\mu}, \sigma^{2} \mathbf{I}_{p}\right)$, and let $\widehat{\mathbf{a}} \triangleq \operatorname{vec}(\widehat{\mathbf{A}})$ be the solution to (4) with $q=1$. Then we have $\mathrm{df} \triangleq \operatorname{tr}\left(\frac{\partial \hat{\mathrm{a}}}{\partial \mathrm{x}}\right)$ is of the form

$$
\mathrm{df}_{1}=\operatorname{tr}\left(\left[\mathbf{I}+\gamma_{2} \mathbf{P}_{1} \sum_{s \in \mathcal{B}_{12}}\left(\frac{\mathbf{D}_{s}^{\mathrm{T}} \mathbf{D}_{s}}{\left\|\mathbf{D}_{s} \hat{\mathbf{a}}\right\|_{2}}-\frac{\mathbf{D}_{s}^{\mathrm{T}} \mathbf{D}_{s} \widehat{\mathbf{a}} \widehat{\mathbf{a}}^{\mathrm{T}} \mathbf{D}_{s}^{\mathrm{T}} \mathbf{D}_{s}}{\left\|\mathbf{D}_{s} \widehat{\mathbf{a}}\right\|_{2}^{3}}\right)\right]^{-1} \mathbf{P}_{1}\right),
$$

where $\mathbf{D}_{s}$ and $\mathbf{P}_{1}$ are defined in $(S .13)$ and $(S .14)$, respectively. 
Following a similar proof technique, we provide an unbiased estimator for the degrees of freedom of the sparse convex clustering with $q=2$.

Lemma S.4 Assume $X_{i} . \stackrel{i i d}{\sim} M V N_{p}\left(\boldsymbol{\mu}, \sigma^{2} \mathbf{I}_{p}\right)$, and let $\widehat{\mathbf{a}}$ be the solution to 4 with $q=2$. Therefore, the degrees of freedom is

$$
\begin{aligned}
\mathrm{df}_{2}= & \operatorname{tr}\left(\left[\mathbf{I}+\gamma_{1} \mathbf{P}_{2} \sum_{s \in \hat{\mathcal{B}}_{2} \cap\{1, \ldots,|\mathcal{E}|\}}\left(\frac{\mathbf{D}_{s}^{\mathrm{T}} \mathbf{D}_{s}}{\left\|\mathbf{D}_{s} \hat{\mathbf{a}}\right\|_{2}}-\frac{\mathbf{D}_{s}^{\mathrm{T}} \mathbf{D}_{s} \widehat{\mathbf{a}} \hat{\mathbf{a}}^{\mathrm{T}} \mathbf{D}_{s}^{\mathrm{T}} \mathbf{D}_{s}}{\left\|\mathbf{D}_{s} \hat{\mathbf{a}}\right\|_{2}^{3}}\right)\right.\right. \\
& \left.\left.+\gamma_{2} \mathbf{P}_{2} \sum_{s \in \hat{\mathcal{B}}_{2} \cap\{|\mathcal{E}|+1, \ldots,|\mathcal{E}|+p\}}\left(\frac{\mathbf{D}_{s}^{\mathrm{T}} \mathbf{D}_{s}}{\left\|\mathbf{D}_{s} \hat{\mathbf{a}}\right\|_{2}}-\frac{\mathbf{D}_{s}^{\mathrm{T}} \mathbf{D}_{s} \hat{\mathbf{a}} \hat{\mathbf{a}} \hat{\mathbf{D}}_{s}^{\mathrm{T}} \mathbf{D}_{s}}{\left\|\mathbf{D}_{s} \hat{\mathbf{a}}\right\|_{2}^{3}}\right)\right]^{-1} \mathbf{P}_{2}\right) .
\end{aligned}
$$

Proofs of Lemmas S.3 $\mid$ S.4; Following the arguments in Tan and Witten (2015), the number of degrees of freedom (df) of (4), when $q=1$ or 2 , can be derived under the assumption $w_{l}=1, l \in \mathcal{E}$ and $X_{i}$. $\sim \operatorname{MVN}_{p}\left(\boldsymbol{\mu}, \sigma^{2} \mathbf{I}_{p}\right)$.

Case $q=1$ : Rewrite (4) into the following formulation:

$$
\min _{\mathbf{a} \in \mathbb{R}^{n p \times 1}} \frac{1}{2}\|\mathbf{x}-\mathbf{a}\|_{2}^{2}+\gamma_{1} \sum_{l \in \mathcal{E}} w_{l}\left\|\mathbf{C}_{l} \mathbf{a}\right\|_{1}+\gamma_{2} \sum_{j=1}^{p} u_{j}\left\|\left(\mathbf{e}_{j}^{* T} \otimes \mathbf{I}_{n}\right) \mathbf{a}\right\|_{2},
$$

where $\mathbf{e}_{j}^{*}$ is a $p$-dim vector with its $j$-th element as 1 and 0 otherwise.

Define

$$
\mathbf{D}_{j}=\left\{\begin{array}{ll}
w_{j} \mathbf{C}_{j} & \text { if } s=1, \ldots,|\mathcal{E}| \\
u_{s-|\mathcal{E}|}\left(\mathbf{e}_{s-|\mathcal{E}|}^{* t} \otimes \mathbf{I}_{n}\right), & \text { if } s=|\mathcal{E}|+1, \ldots,|\mathcal{E}|+p
\end{array},\right.
$$

and let $\mathbf{D}^{\mathrm{T}}=\left(\mathbf{D}_{1}^{\mathrm{T}}, \ldots, \mathbf{D}_{|\mathcal{E}|+p}^{\mathrm{T}}\right)$. Then $(\mathrm{S} .12)$ becomes

$$
\min _{\mathbf{a} \in \mathbb{R}^{n p \times 1}} \frac{1}{2}\|\mathbf{x}-\mathbf{a}\|_{2}^{2}+\gamma_{1} \sum_{s=1}^{|\mathcal{E}|}\left\|\mathbf{D}_{s} \mathbf{a}\right\|_{1}+\gamma_{2} \sum_{s=|\mathcal{E}|+1}^{|\mathcal{E}|+p}\left\|\mathbf{D}_{s} \mathbf{a}\right\|_{2}
$$

Actually, the second term can be written component-wisely into $\gamma_{1} \sum_{s=1}^{|\mathcal{E}|} \sum_{j=1}^{p}\left|\mathbf{d}_{s j}^{\mathrm{T}} \mathbf{a}\right|$, where $\mathbf{d}_{s j}$ is the vector consisting of the $j$-th row of $\mathbf{D}_{s}$.

Let $\widehat{\mathcal{B}}_{1}=\widehat{\mathcal{B}}_{11} \bigcup \widehat{\mathcal{B}}_{12}$, where $\widehat{\mathcal{B}}_{11}=\left\{(s, j):\left|\mathbf{d}_{s j}^{\mathrm{T}} \hat{\mathbf{a}}\right| \neq 0, s=1, \ldots,|\mathcal{E}|, j=1, \ldots, p\right\}$ and $\widehat{\mathcal{B}}_{12}=\left\{s:\left\|\mathbf{D}_{s} \widehat{\mathbf{a}}\right\|_{2} \neq 0, s=|\mathcal{E}|+1, \ldots,|\mathcal{E}|+p\right\}$. The derivative of $(\mathbf{S} .12)$ is obtained as

$$
\mathbf{x}-\mathbf{a}=\gamma_{1} \sum_{s=1}^{|\mathcal{E}|} \sum_{j=1}^{p} f_{s j} \mathbf{d}_{s j}+\gamma_{2} \sum_{s=|\mathcal{E}|+1}^{|\mathcal{E}|+p} \mathbf{D}_{s}^{\mathrm{T}} \mathbf{g}_{s}
$$


where $f_{s j}=\operatorname{sgn}\left(\mathbf{d}_{s j}^{\mathrm{T}} \widehat{\mathbf{a}}\right)$, if $(s, j) \in \widehat{\mathcal{B}}_{11}$ and $f_{s j} \in[-1,1]$, if $s \notin \widehat{\mathcal{B}}_{11}$, and $\mathbf{g}_{s}=\mathbf{D}_{s} \widehat{\mathbf{a}} /\left\|\mathbf{D}_{s} \widehat{\mathbf{a}}\right\|_{2}$, if $s \in \widehat{\mathcal{B}}_{12}$ and $\mathbf{g}_{s} \in\left\{\Gamma:\|\Gamma\|_{2} \leqslant 1\right\}$, if $s \notin \widehat{\mathcal{B}}_{12}$.

Define matrix $\mathbf{D}_{-\hat{\mathcal{B}}_{1}}$ by removing the rows of $\mathbf{D}$ corresponding to those elements in $\widehat{\mathcal{B}}_{1}$, and

$$
\mathbf{P}_{1}=\mathbf{I}-\mathbf{D}_{-\hat{\mathcal{B}}_{1}}^{\mathrm{T}}\left(\mathbf{D}_{-\hat{\mathcal{B}}_{1}} \mathbf{D}_{-\hat{\mathcal{B}}_{1}}^{\mathrm{T}}\right)^{+} \mathbf{D}_{-\hat{\mathcal{B}}_{1}}
$$

By the property $\mathrm{D}_{-\widehat{\mathcal{B}}_{1}} \widehat{\mathbf{a}}=\mathbf{0}$,

$$
\begin{aligned}
\mathbf{P}_{1} \mathbf{x}-\widehat{\mathbf{a}} & =\gamma_{1} \mathbf{P}_{1} \sum_{s=1}^{|\mathcal{E}|} \sum_{j=1}^{p} f_{s j} \mathbf{d}_{s j}+\gamma_{2} \mathbf{P}_{1} \sum_{s=|\mathcal{E}|+1}^{|\mathcal{E}|+p} \mathbf{D}_{s}^{\mathrm{T}} \mathbf{g}_{s} \\
& =\gamma_{1} \mathbf{P}_{1} \sum_{(s, j) \in \mathcal{B}_{11}} \operatorname{sgn}\left(\mathbf{d}_{s j}^{\mathrm{T}} \hat{\mathbf{a}}\right) \mathbf{d}_{s j}+\gamma_{2} \mathbf{P}_{1} \sum_{s \in \mathcal{B}_{12}} \frac{\mathbf{D}_{s}^{\mathrm{T}} \mathbf{D}_{s} \hat{\mathbf{a}}}{\left\|\mathbf{D}_{s} \widehat{\mathbf{a}}\right\|_{2}}
\end{aligned}
$$

By the property shown by Vaiter et al. (2012); i.e., there exists a neighborhood around almost every $\mathbf{x}$ such that the solution $\widehat{\mathbf{a}}$ is locally constant with respect to $\mathbf{x}$, the derivative of the above equation with respect to $\mathrm{x}$ is

$$
\mathbf{P}_{1}-\frac{\partial \hat{\mathbf{a}}}{\partial \mathbf{x}}=\gamma_{2} \mathbf{P}_{1} \sum_{s \in \mathcal{B}_{12}}\left(\frac{\mathbf{D}_{s}^{\mathrm{T}} \mathbf{D}_{s}}{\left\|\mathbf{D}_{s} \hat{\mathbf{a}}\right\|_{2}}-\frac{\mathbf{D}_{s}^{\mathrm{T}} \mathbf{D}_{s} \widehat{\mathbf{a}} \hat{\mathbf{a}}^{\mathrm{T}} \mathbf{D}_{s}^{\mathrm{T}} \mathbf{D}_{s}}{\left\|\mathbf{D}_{s} \hat{\mathbf{a}}\right\|_{2}^{3}}\right) \frac{\partial \widehat{\mathbf{a}}}{\partial \mathbf{x}}
$$

Therefore, $\mathrm{df} \triangleq \operatorname{tr}\left(\frac{\partial \hat{\mathbf{a}}}{\partial \mathbf{x}}\right)$ is of the form

$$
\mathrm{df}_{1}=\operatorname{tr}\left(\left[\mathbf{I}+\gamma_{2} \mathbf{P}_{1} \sum_{s \in \mathcal{B}_{12}}\left(\frac{\mathbf{D}_{s}^{\mathrm{T}} \mathbf{D}_{s}}{\left\|\mathbf{D}_{s} \widehat{\mathbf{a}}\right\|_{2}}-\frac{\mathbf{D}_{s}^{\mathrm{T}} \mathbf{D}_{s} \widehat{\mathbf{a}} \hat{\mathbf{a}}^{\mathrm{T}} \mathbf{D}_{s}^{\mathrm{T}} \mathbf{D}_{s}}{\left\|\mathbf{D}_{s} \hat{\mathbf{a}}\right\|_{2}^{3}}\right)\right]^{-1} \mathbf{P}_{1}\right)
$$

Case $q=2$ : Rewrite (4) into the following form when $q=2$, the $L_{2}$-norm:

$$
\min _{\mathbf{a} \in \mathbb{R}^{n p \times 1}} \frac{1}{2}\|\mathbf{x}-\mathbf{a}\|_{2}^{2}+\gamma_{1} \sum_{j=1}^{|\mathcal{E}|}\left\|\mathbf{D}_{s} \mathbf{a}\right\|_{2}+\gamma_{2} \sum_{s=|\mathcal{E}|+1}^{|\mathcal{E}|+p}\left\|\mathbf{D}_{s} \mathbf{a}\right\|_{2} .
$$

Let $\widehat{\mathcal{B}}_{2}=\left\{s:\left\|\mathbf{D}_{s} \widehat{\mathbf{a}}\right\|_{2} \neq 0, s=1, \ldots,|\mathcal{E}|+p\right\}$. The derivative of $(\mathbf{S} .15)$ is obtained as

$$
\mathbf{x}-\mathbf{a}=\gamma_{1} \sum_{s=1}^{|\mathcal{E}|} \mathbf{D}_{s}^{\mathrm{T}} \mathbf{g}_{j}+\gamma_{2} \sum_{s=|\mathcal{E}|+1}^{|\mathcal{E}|+p} \mathbf{D}_{s}^{\mathrm{T}} \mathbf{g}_{j}
$$


where $\mathbf{g}_{s}=\mathbf{D}_{s} \hat{\mathbf{a}} /\left\|\mathbf{D}_{s} \hat{\mathbf{a}}\right\|_{2}$, if $s \in \widehat{\mathcal{B}}_{2}$ and $\mathbf{g}_{s} \in\left\{\Gamma:\|\Gamma\|_{2} \leqslant 1\right\}$, if $s \notin \widehat{\mathcal{B}}_{2}$. Then, define matrix $\mathbf{D}_{-\widehat{\mathcal{B}}_{2}}$ by removing the rows of $\mathbf{D}$ corresponding to those elements in $\widehat{\mathcal{B}}_{2}$, and $\mathbf{P}_{2}=\mathbf{I}-\mathbf{D}_{-\hat{\mathcal{B}}_{2}}^{\mathrm{T}}\left(\mathbf{D}_{-\hat{\mathcal{B}}_{2}} \mathbf{D}_{-\hat{\mathcal{B}}_{2}}^{\mathrm{T}}\right)^{+} \mathbf{D}_{-\widehat{\mathcal{B}}_{2}}$. It follows

$$
\begin{aligned}
\frac{\partial \widehat{\mathbf{a}}}{\partial \mathbf{x}}= & {\left[\mathbf{I}+\gamma_{1} \mathbf{P}_{2} \sum_{s \in \hat{\mathcal{B}}_{2} \cap\{1, \ldots,|\mathcal{E}|\}}\left(\frac{\mathbf{D}_{s}^{\mathrm{T}} \mathbf{D}_{s}}{\left\|\mathbf{D}_{s} \hat{\mathbf{a}}\right\|_{2}}-\frac{\mathbf{D}_{s}^{\mathrm{T}} \mathbf{D}_{s} \hat{\mathbf{a}} \widehat{\mathbf{a}}^{\mathrm{T}} \mathbf{D}_{s}^{\mathrm{T}} \mathbf{D}_{s}}{\left\|\mathbf{D}_{s} \hat{\mathbf{a}}\right\|_{2}^{3}}\right)\right.} \\
& \left.+\gamma_{2} \mathbf{P}_{2} \sum_{s \in \hat{\mathcal{B}}_{2} \cap\{|\mathcal{E}|+1, \ldots,|\mathcal{E}|+p\}}\left(\frac{\mathbf{D}_{s}^{\mathrm{T}} \mathbf{D}_{s}}{\left\|\mathbf{D}_{s} \hat{\mathbf{a}}\right\|_{2}}-\frac{\mathbf{D}_{s}^{\mathrm{T}} \mathbf{D}_{s} \hat{\mathbf{a}} \widehat{\mathbf{a}}^{\mathrm{T}} \mathbf{D}_{s}^{\mathrm{T}} \mathbf{D}_{s}}{\left\|\mathbf{D}_{s} \hat{\mathbf{a}}\right\|_{2}^{3}}\right)\right]^{-1} \mathbf{P}_{2} .
\end{aligned}
$$

Therefore, the df when $q=2$ is of the form

$$
\begin{aligned}
\mathrm{df}_{2}= & \operatorname{tr}\left(\left[\mathbf{I}+\gamma_{1} \mathbf{P}_{2} \sum_{s \in \hat{\mathcal{B}}_{2} \cap\{1, \ldots,|\mathcal{E}|\}}\left(\frac{\mathbf{D}_{s}^{\mathrm{T}} \mathbf{D}_{s}}{\left\|\mathbf{D}_{s} \hat{\mathbf{a}}\right\|_{2}}-\frac{\mathbf{D}_{s}^{\mathrm{T}} \mathbf{D}_{s} \hat{\mathbf{a}} \hat{\mathbf{a}}^{\mathrm{T}} \mathbf{D}_{s}^{\mathrm{T}} \mathbf{D}_{s}}{\left\|\mathbf{D}_{s} \hat{\mathbf{a}}\right\|_{2}^{3}}\right)\right.\right. \\
& \left.\left.+\gamma_{2} \mathbf{P}_{2} \sum_{s \in \hat{\mathcal{B}}_{2} \cap\{|\mathcal{E}|+1, \ldots,|\mathcal{E}|+p\}}\left(\frac{\mathbf{D}_{s}^{\mathrm{T}} \mathbf{D}_{s}}{\left\|\mathbf{D}_{s} \hat{\mathbf{a}}\right\|_{2}}-\frac{\mathbf{D}_{s}^{\mathrm{T}} \mathbf{D}_{s} \hat{\mathbf{a}} \hat{\mathbf{a}} \mathbf{D}_{s}^{\mathrm{T}} \mathbf{D}_{s}}{\left\|\mathbf{D}_{s} \hat{\mathbf{a}}\right\|_{2}^{3}}\right)\right]^{-1} \mathbf{P}_{2}\right) .
\end{aligned}
$$

